\title{
Spatio-Temporal Modeling of the Optical Properties of VCSELs in the Presence of Polarization Effects
}

\author{
Josep Mulet and Salvador Balle
}

\begin{abstract}
In this paper, we develop an optical dynamical model for vertical-cavity surface-emitting lasers (VCSELs) which describes, in an unified way, polarization and spatial effects. The model is based on equations for the lateral dependence of the slowly-varying amplitudes of the optical field in both circular polarizations, and equations for the carrier density in both spin orientations. This provides a natural generalization of the Spin Flip Model for the description of polarization properties of VCSELs extensively used in the literature. In its present form, the model assumes given functional dependence of the guiding mechanisms (built-in refractive index and thermal lensing) as well as the spatial dependence of the current density.

We investigate the transverse mode behavior of gain-guided, bottom and top-emitter VCSELs by implementing the model with an analytical approximation to the susceptibility of quantum-well semiconductors. We demonstrate that the stronger the thermal lens, the stronger the tendency toward multimode operation, which indicates that high lateral uniformity of the temperature is required in order to maintain single mode operation in gain-guided VCSELs. We perform analytical calculations of the threshold curves in both types of VCSELs. Also, close-to-threshold numerical simulations show that, depending on the current shape, thermal lensing strength and relative detuning, different transverse modes can be selected.
\end{abstract}

Index Terms-Laser modes, modeling, polarization, semiconductor lasers, surface-emitting lasers.

\section{INTRODUCTION}

V ERTICAL-CAVITY surface-emitting lasers (VCSELs) are promising devices for many optical applications and are particularly of interest because of their single-longitudinal mode emission, easy integration in 2-D arrays, and narrow circular output beams. However, relatively large apertures are required in order to obtain substantial optical power, which lead to multitransverse mode behavior of the VCSEL [1]. In addition, the VCSEL's cavity lacks a mechanism effective to pin the polarization of the optical field. Therefore, light-polarization instabilities are often observed when the current is increased [2], as well as excitation of higher order transverse modes. Since many applications require stability in both the emission profile and polarization, it is important to design the VCSEL appropriately. This fact motivates the study, characterization and control of polarization and transverse mode dynamics.

Manuscript received September 12, 2001; revised November 13, 2001 This work was supported by the European Commission under the VISTA HP-TRN and the Spanish MCyT under Projects TIC99-0645-C05-02 and BFM2000-1108.

The authors are with the Instituto Mediterráneo de Estudios Avanzados (IMEDEA), Consejo Superior de Investigaciones Científicas, Universitat de les Illes Balears, Campus UIB, E-07071 Palma de Mallorca, Spain (e-mail: mulet@imedea.uib.es).

Publisher Item Identifier S 0018-9197(02)01761-X.
Most studies of polarization dynamics have been concerned with devices where the spatial degrees of freedom can be disregarded. A first explanation for the observed polarization dynamics and instabilities was put forward by Choquette and coworkers [3], [4]. Their main argument is that, due to residual cavity anisotropies, linearly polarized modes experience different net modal gains, so that at threshold the mode with larger gain (usually that closest to the gain peak) is selected. However, as the current is increased the temperature of device also increases, leading to a redshift of the gain curve relative to the linearly polarized modes that may cause a polarization switch from the high-frequency mode to the low-frequency mode. This model has been further extended to account for the effects of thermal lensing, gain-dispersion, and temperature-dependent free-carrier absorption [5], [6]. A different kind of explanation for polarization switching in single-mode devices is given by the so-called Spin-Flip Model (SFM) [7], which is based on a generalization of the gas laser theory to the magnetic sublevels of the conduction and heavy-hole bands in a quantum well (QW). The SFM explains the polarization switching in VCSELs as the result of an instability of the phase locking among the circularly polarized components of the optical field that arises from the coupling between amplitude and phase due to the linewidth enhancement factor. The SFM has been extensively applied to analyze the polarization selection and instabilities: the interplay of linear and nonlinear cavity anisotropies in polarization switching, the influence of magnetic fields [2], mode hopping, and the polarization resolved intensity noise [8]. Recently, the SFM has been justified from a microscopic point of view [9] and it has also been extended for including the frequency-dependence of the carrier-induced gain and refractive index, showing that the thermal mechanism discussed before and that coming from phase instabilities can coexist depending on the VCSEL characteristics [10], [11].

On the other hand, several methods have been devised recently to analyze the cavity modes of VCSELs in a scalar, semi-vectorial, or fully-vectorial description [12]-[16]. These methods are able to determine the modal frequencies, profiles, and threshold gains from the distribution of the index of refraction associated with a given device structure. However, they cannot be directly applied to the study of spatio-temporal dynamics of the system because these methods are passive and static, so that they disregard the coupling of the optical field with the carrier density and the associated index change through spatial hole burning, which has been found to be relevant in VCSELs despite the presence of other guiding mechanisms [17]. Moreover, the index of refraction of the materials defining the cavity is temperature dependent; hence, the refractive index distribution changes as the current is 
increased due to device self-heating through Joule heat dissipation. Heat is mainly generated close to the cavity axis, so a radial profile of temperature develops with higher temperatures close to the cavity axis. As a consequence, besides a global increase of the average index that is responsible for the observed redshift of the cavity modes, the refractive index is higher at the center of the device than in the outer regions, a phenomenon known as thermal lensing (TL). The effects of TL are usually weak, since the thermal rate of change in the index of refraction is of the order of $|\partial n / \partial T|_{\lambda} \sim 5 \times 10^{-4} K^{-1}$. Nevertheless, TL can strongly influence the transverse mode properties in weakly index-guided lasers-such as oxidized VCSELs with the oxide layer placed close to a field node- or purely gain-guided diode lasers which do not possess any built-in index waveguide and where lateral confinement of the optical field occurs only via a combination of gain-guiding and index anti-guiding mechanisms [17], [18].

In order to model the dynamics of VCSELs with spatial degrees of freedom, a modal expansion of the electric field is often used [19], [20], thereby including spatial holeburning effects. However, a drawback of such an approach is that the number and type of modes considered in the description has to be fixed and determined a priori, and usually only a few low-order modes are included. In relatively large VCSELs that support several transverse modes, some of which may have quite similar frequencies, it is preferable to directly investigate the spatio-temporal dynamics of the optical field, either considering [21], [22], or not considering [23], [24] the polarization of the optical field. The direct inclusion of transverse effects in the dynamics of multimode VCSELs requires to consider the frequency dependence of both the gain and refractive index of the material that constitutes the active region. In addition, they should also correctly incorporate the nonlinear dependence on the carrier density because of the inhomogeneous carrier distribution arising from the localized injected current. The most natural way to incorporate both the gain and refractive index is through the optical susceptibility of the active region, which could be obtained in either a microscopic [25]-[29] or mesoscopic framework [30]-[32]. The former gives a very accurate description but requires a huge computational effort; the latter, despite approximations, can provide an accurate description of the active medium and can be included directly into the laser dynamics [11], [29], [33].

This paper is organized as follows. In Section II, we present a detailed description of the optical VCSEL model implemented in this paper, which generalizes the SFM in order to include: 1) the spatial dependence of both the field and carrier densities and 2) a susceptibility tensor that describes the frequency-dependence of the gain and refractive index distributions induced by the carriers. In Section III, we present an analytical approach to obtain the threshold of transverse modes. In Section IV, we discuss the results of numerical simulations. Finally, Section V is devoted to summarizing and concluding our paper.

\section{MODEL}

In weakly-index guided or purely gain-guided devices, the optical field inside the VCSEL cavity can be considered as almost totally polarized in the transverse plane to the cavity axis.

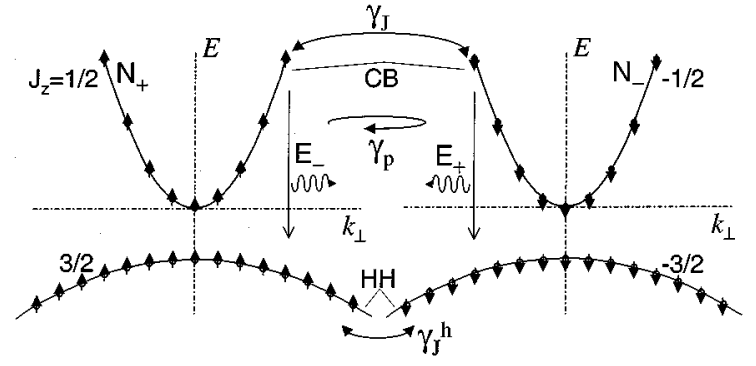

Fig. 1. Scheme of the allowed transitions in the spin subbands of a strained QW, $(\mathrm{HH})$ heavy hole with $J_{z}= \pm 3 / 2$, and (CB) conduction bands with $J_{z}=$ $\pm 1 / 2$. Electrons with opposite spin are mixed at rate $\gamma_{J}$. Emitted photons with opposite circular polarization $\left(\mathrm{E}_{ \pm}\right)$are coupled through the linear birefringence $\gamma_{p}$

In a system with perfect cylindrical symmetry, any linearly polarized state of the optical field is allowed. In crystals with cubic symmetry, this rotational invariance is not perfectly preserved. Moreover, the VCSEL cavity has weak optical anisotropies (due to either residual strain incorporated during device processing or to other sources as the elasto-optic [34] or electro-optic effects [35]) that select two preferred orthogonal orientations for the optical field, $\hat{x}$ and $\hat{y}$, which usually correspond to the underlying crystallographic axes. We assume that the preferred orientations are the same in all epitaxial layers defining the VCSEL cavity; hence, the optical susceptibility of the passive cavity is diagonal in the basis of linearly polarized states. However, the polarization state of the optical field emitted by the VCSEL also depends on its interaction with the active region's material, governed by the selection rules of quantum mechanics. In crystals with cubic symmetry, and when the optical field propagates along the quantization axes of the crystal $(\hat{z})$, the selection rules for the transitions impose, among others, the conservation of the third component of the angular momentum. The optical susceptibility of the active region is, therefore, diagonal in the basis of circularly polarized states of the optical field; hence, we switch to it due to the resulting simplified description of the dynamical interaction with the active material. It is then also natural to distinguish between spin-up and spin-down electrons and holes, since they couple to optical transitions with opposite circular polarization. In addition, spin-up and spin-down carriers are coupled among them through spin-flip mechanisms that may reverse the particle's spin [36], and which we shall describe through an effective spin relaxation rate. These processes are graphically sketched in Fig. 1 for the case of only one conduction band for the electrons and one heavy-hole band. In Section II-A, we discuss the optical part of the model, while in Section II-B, we treat the evolution of the carrier densities.

\section{A. Optical Model}

In this subsection, we detail the procedure outlined above in order to obtain our dynamical model for the VCSEL taking into account both the polarization and transverse degrees of freedom. We start from Maxwell's equations in the frequency domain, and after determining the optical carrier frequency of the VCSEL emission, we return to the time domain in order to find the dynamical equations for the slowly varying amplitudes (SVA) of the circularly polarized optical-field components. 
From Maxwell's equations in the frequency domain, the distribution of each linearly polarized component of the optical field $\mathcal{E}_{k}(\vec{r} ; \omega)$ (with $k=\hat{x}, \hat{y}$ ) is given by

$$
\begin{aligned}
& \left\{\nabla_{\perp}^{2}+\partial_{z}^{2}+\frac{\omega^{2}}{c^{2}}\left[1+\chi_{b}^{k}(\vec{r} ; \omega)\right]\right\} \mathcal{E}_{k}(\vec{r} ; \omega) \\
& =-\frac{\omega^{2}}{c^{2}}\left\{\frac{\mathcal{P}_{k}(\vec{r} ; \omega)}{\varepsilon_{0}}-\chi_{b}^{k}(\vec{r} ; \omega) \mathcal{E}_{k}(\vec{r} ; \omega)\right\} R_{W}\left(z, z_{0}\right)
\end{aligned}
$$

where $\nabla_{\perp}^{2}=\partial_{x}^{2}+\partial_{y}^{2}$ and $\chi_{b}^{k}(\vec{r} ; \omega)$ are the frequency-dependent susceptibility distribution of the passive material filling the cavity for a field polarized along the $k$-direction, $\mathcal{P}_{k}(\vec{r} ; \omega)$ is the $k$-component of the material dipole density due to the active material, thus providing both gain and a change in refraction index, $R_{W}\left(z, z_{0}\right)$ is the rectangle function, which is 1 if $z_{0}<z<z_{0}+W$ and zero otherwise, that specifies the position of the active region, which we consider to be made of a single QW whose thickness is $W$. In the case of multiple QWs, we assume that they are all in the same electrical state, so that the total thickness of the active region is scaled by the number of wells.

By considering that due to the short cavity length $L$, the VCSEL supports a single longitudinal mode in the vicinity of the gain maximum, the optical-field components can be split into their longitudinal and transverse parts

$$
\begin{aligned}
\mathcal{E}_{k}(\vec{r} ; \omega) & =\left(e^{i q_{k} z}+\frac{e^{-i q_{k} z}}{r_{1}^{k}}\right) \mathcal{A}_{k}\left(\vec{r}_{\perp} ; \omega\right) \\
& \equiv f_{k}(z) \mathcal{A}_{k}\left(\vec{r}_{\perp} ; \omega\right)
\end{aligned}
$$

where $\vec{r}_{\perp}=(x, y)$. The longitudinal modes of the VCSEL cavity are determined by the round-trip condition in the plane-wave approximation. For a linearly polarized wave, it reads

$$
r_{1}^{k} r_{2}^{k} e^{2 i q_{k} L}=1
$$

where $q_{k}$ stands for the complex propagation constant of the longitudinal mode linearly polarized along the $k$-direction, with its real and imaginary parts determining the wavelength and threshold gain for this mode, and $L$ is the physical cavity length corresponding to the separation between the two Bragg mirrors. $r_{1}^{k}$ and $r_{2}^{k}$ denote the frequency-dependent amplitude reflectivities of the top and bottom Bragg reflectors. Moreover, Bragg mirrors are usually birefringent, displaying polarizationdependent reflectivities $r_{1,2}^{x} \neq r_{1,2}^{y}$. This effect provides different propagation constants and, in general, different longitudinal profiles for the two linearly polarized modes.

Upon substitution of (2) into (1) and by projecting onto the longitudinal mode $f_{k}(z)$, the transverse-field distributions in the cavity section $\mathcal{A}_{k}\left(\vec{r}_{\perp} ; \omega\right)$ are given by

$$
\begin{aligned}
\left\{\nabla_{\perp}^{2}+\frac{\omega^{2}}{c^{2}}\left[1+\chi_{e}^{k}\left(\vec{r}_{\perp} ; \omega\right)\right]-q_{k}^{2}\right\} & \mathcal{A}_{k}\left(\vec{r}_{\perp} ; \omega\right) \\
& =-\frac{\omega^{2}}{\varepsilon_{0} c^{2}} \mathcal{B}_{k}\left(\vec{r}_{\perp} ; \omega\right)
\end{aligned}
$$

where we have defined

$$
\begin{aligned}
F_{k}(z)= & \frac{e^{i q_{k} z}+\frac{e^{-i q_{k} z}}{r_{1}^{k}}}{\sqrt{\int_{0}^{L} d z\left|e^{i q_{k} z}+\frac{e^{-i q_{k} z}}{r_{1}^{k}}\right|^{2}}} \\
\chi_{e}^{k}\left(\vec{r}_{\perp} ; \omega\right)= & \int_{0}^{L} d z \chi_{b}^{k}(\vec{r} ; \omega)\left|F_{k}(z)\right|^{2} \\
& -\int_{z_{0}}^{z_{0}+W} d z \chi_{b}^{k}(\vec{r} ; \omega)\left|F_{k}(z)\right|^{2} \\
\mathcal{B}_{k}\left(\vec{r}_{\perp} ; \omega\right)= & \int_{z_{0}}^{z_{0}+W} d z \mathcal{P}_{k}(\vec{r} ; \omega) F_{k}^{*}(z) .
\end{aligned}
$$

where

$F_{k}(z)$ normalized longitudinal-field profile;

$\chi_{e}^{k} \quad$ longitudinal average of the passive material's susceptibility;

$\mathcal{B}_{k} \quad$ projection of the active material's dipole density onto the corresponding $k$-component of the longitudinal mode.

As already commented, in our system the selection rules for the optical transitions impose the conservation of the axial component of the angular momentum, hence the interaction with the active material is diagonal in the basis of circularly polarized optical states. Therefore we express the optical fields in the basis of left- and right-circularly polarized components

$$
\mathcal{A}_{ \pm}=\frac{\mathcal{A}_{x} \pm i \mathcal{A}_{y}}{\sqrt{2}}
$$

where the optical interaction with the QW-based active region is naturally expressed. Then, (4) reads

$$
\begin{aligned}
\left\{\nabla_{\perp}^{2}\right. & \left.+\frac{\omega^{2}}{c^{2}}\left[1+\chi_{e}\left(\vec{r}_{\perp} ; \omega\right)\right]-q^{2}\right\} \mathcal{A}_{ \pm}\left(\vec{r}_{\perp} ; \omega\right) \\
& +\left[\frac{\omega^{2}}{c^{2}} \delta \chi_{e}\left(\vec{r}_{\perp} ; \omega\right)-\delta q^{2}\right] \mathcal{A}_{\mp}\left(\vec{r}_{\perp} ; \omega\right) \\
= & -\frac{\omega^{2}}{\varepsilon_{0} c^{2}} \mathcal{B}_{ \pm}\left(\vec{r}_{\perp} ; \omega\right)
\end{aligned}
$$

where we have defined

$$
\begin{aligned}
\chi_{e}\left(\vec{r}_{\perp} ; \omega\right) & =\frac{\chi_{e}^{x}\left(\vec{r}_{\perp} ; \omega\right)+\chi_{e}^{y}\left(\vec{r}_{\perp} ; \omega\right)}{2} \\
\delta \chi_{e}\left(\vec{r}_{\perp} ; \omega\right) & =\frac{\chi_{e}^{x}\left(\vec{r}_{\perp} ; \omega\right)-\chi_{e}^{y}\left(\vec{r}_{\perp} ; \omega\right)}{2} \\
q^{2} & =\frac{q_{x}^{2}+q_{y}^{2}}{2} \\
\delta q^{2} & =\frac{q_{x}^{2}-q_{y}^{2}}{2} \\
\mathcal{B}_{ \pm}\left(\vec{r}_{\perp} ; \omega\right) & =\frac{\mathcal{B}_{x}\left(\vec{r}_{\perp} ; \omega\right) \pm i \mathcal{B}_{y}\left(\vec{r}_{\perp} ; \omega\right)}{\sqrt{2}} .
\end{aligned}
$$


Given the QW's susceptibility components in the circular basis, $\mathcal{P}_{ \pm}=\varepsilon_{0} \chi_{ \pm}(\vec{r} ; \omega) \mathcal{E}_{ \pm}$, the linear components of the material dipole density can be expressed as

$$
\begin{aligned}
\mathcal{P}_{x}\left(\vec{r}_{\perp}, z ; \omega\right)=\varepsilon_{0}\left[\frac{\chi_{+}+\chi_{-}}{2} f_{x}(z) \mathcal{A}_{x}\left(\vec{r}_{\perp} ; \omega\right)\right. \\
\left.\quad-\frac{\chi_{+}-\chi_{-}}{2 i} f_{y}(z) \mathcal{A}_{y}\left(\vec{r}_{\perp} ; \omega\right)\right], \\
\mathcal{P}_{y}\left(\vec{r}_{\perp}, z ; \omega\right)=\varepsilon_{0}\left[\begin{array}{l}
\frac{\chi_{+}-\chi_{-}}{2 i} f_{x}(z) \mathcal{A}_{x}\left(\vec{r}_{\perp} ; \omega\right) \\
\left.+\frac{\chi_{+}+\chi-}{2} f_{y}(z) \mathcal{A}_{y}\left(\vec{r}_{\perp} ; \omega\right)\right]
\end{array}\right.
\end{aligned}
$$

and, upon substituting the above expressions into (7) and (14), we obtain

$$
\mathcal{B}_{ \pm}=\varepsilon_{0}\left(\mathcal{A}_{+} S_{ \pm}+\mathcal{A}_{-} T_{ \pm}\right)
$$

where

$$
\begin{aligned}
S_{ \pm}= & \int_{z_{0}}^{z_{0}+W} d z\left[\frac{\chi_{+}+\chi_{-}}{4}\left(\left|F_{x}(z)\right|^{2} \pm\left|F_{y}(z)\right|^{2}\right)\right. \\
& \left.+\frac{\chi_{+}-\chi_{-}}{4}\left[\frac{f_{x}^{*}(z) f_{y}(z)}{\int_{0}^{L} d z\left|f_{x}(z)\right|^{2}} \pm \frac{f_{x}(z) f_{y}^{*}(z)}{\int_{0}^{L} d z\left|f_{y}(z)\right|^{2}}\right)\right] \\
T_{ \pm}= & \int_{z_{0}}^{z_{0}+W} d z\left[\frac{\chi_{+}+\chi_{-}}{4}\left(\left|F_{x}(z)\right|^{2} \mp\left|F_{y}(z)\right|^{2}\right)\right. \\
& \left.-\frac{\chi_{+}-\chi_{-}}{4}\left[\frac{f_{x}^{*}(z) f_{y}(z)}{\int_{0}^{L} d z\left|f_{x}(z)\right|^{2}} \mp \frac{f_{x}(z) f_{y}^{*}(z)}{\int_{0}^{L} d z\left|f_{y}(z)\right|^{2}}\right)\right] .
\end{aligned}
$$

By splitting the total carrier density inside the QW as $N=N_{+}+N_{-}$, where $N_{ \pm}$stands for the electron density with spin up and down, respectively, the susceptibility components in the circularly polarized basis are $\chi_{ \pm} \equiv \chi_{ \pm}\left(\omega, N_{+}(\vec{r}), N_{-}(\vec{r})\right)$, and they depend on position only through the position dependence of $N_{ \pm}$. Since the QW thickness $W$ is much smaller than the diffusion length, it can be assumed that, inside the QW, the carrier density is almost constant along $z$, i.e., $N_{ \pm}(\vec{r}) \simeq \bar{N}_{ \pm}\left(\vec{r}_{\perp}\right) \equiv(1 / W) \int_{z_{0}}^{z_{0}+W} d z N_{ \pm}(\vec{r})$. Moreover, for weak cavity anisotropies, the longitudinal propagation constants $q_{x}$ and $q_{y}$ are quite similar, so we can consider that the longitudinal mode profiles are almost the same, with $f_{x}(z) \approx f_{y}(z) \equiv f(z)$. In this case, (17) simply reduces to

$$
\mathcal{B}_{ \pm}\left(\vec{r}_{\perp} ; \omega\right)=\varepsilon_{0} \Gamma \chi_{ \pm}\left(\omega, \bar{N}_{+}\left(\vec{r}_{\perp}\right), \bar{N}_{-}\left(\vec{r}_{\perp}\right)\right) \mathcal{A}_{ \pm}\left(\vec{r}_{\perp} ; \omega\right)
$$

where

$$
\Gamma=\frac{\int_{z_{0}}^{z_{0}+W} d z|f(z)|^{2}}{\int_{0}^{L} d z|f(z)|^{2}}
$$

is the longitudinal optical confinement factor that represents the fraction of the intracavity power that is confined to the QW active region. As already noted, in the case of a multiple QW active region, we consider that all the $\mathrm{QWs}$ are identical and in the same state. Hence, we simply scale the single-QW confinement factor by the number of wells. Therefore, (9) becomes

$$
\begin{gathered}
\left\{\nabla_{\perp}^{2}+\frac{\omega^{2}}{c^{2}}\left[1+\chi_{e}\left(\vec{r}_{\perp} ; \omega\right)\right]-q^{2}\right\} \mathcal{A}_{ \pm}\left(\vec{r}_{\perp} ; \omega\right) \\
\quad+\left[\frac{\omega^{2}}{c^{2}} \delta \chi_{e}\left(\vec{r}_{\perp} ; \omega\right)-\delta q^{2}\right] \mathcal{A}_{\mp}\left(\vec{r}_{\perp} ; \omega\right) \\
=-\Gamma \frac{\omega^{2}}{c^{2}} \chi_{ \pm}\left(\omega, \bar{N}_{+}, \bar{N}_{-}\right) \mathcal{A}_{ \pm}\left(\vec{r}_{\perp} ; \omega\right) .
\end{gathered}
$$

By defining

$$
1+\chi_{e}\left(\vec{r}_{\perp} ; \omega\right)=\left[n_{e}(\omega)+i \bar{\alpha}_{i}(\omega)+\Delta n\left(\vec{r}_{\perp} ; \omega\right)\right]^{2}
$$

where

$n_{e}(\omega)$
$\bar{\alpha}_{i}(\omega)$

effective background refractive index experienced by the field in the isotropic, homogeneous cavity;

$\bar{\alpha}_{i}(\omega) \quad \ll n_{e}(\omega)$ - determines the effective absorption in the passive material;

$\Delta n\left(\vec{r}_{\perp} ; \omega\right) \quad$ (small) excess index distribution responsible for the lateral confinement of the optical field.

The excess refractive index distribution $\Delta n\left(\vec{r}_{\perp} ; \omega\right)$ contains all the waveguiding mechanisms present in the device except the carrier-induced refractive index, which is included through the real part of $\chi_{ \pm}$. Hence, in the cavity without anisotropies, we have that the longitudinal mode considered has an optical frequency $\Omega$, determined - from (3), (22), and (23)—by the condition

$$
\frac{\Omega}{c} n_{e}(\Omega)=\operatorname{Re} q(\Omega)
$$

where $\operatorname{Re} q(\Omega)$ stands for the real part of the propagation constant of the longitudinal mode considered. The frequency dependence of $q$ stems from the frequency-dependent reflectivity of the Bragg mirrors, which mainly arises from the reflection phases while within the stopband of the reflectors. Since the indices of the materials forming the passive cavity and the Bragg reflectors are temperature dependent, (24) incorporates the thermal shift of the cavity mode.

Once the longitudinal mode frequency $\Omega$ has been determined, we can tackle the dynamical evolution of the transverse-field profile in the SVA approximation. For the active VCSEL, the optical field is quasi-monochromatic, and we take $\Omega$ as the carrier optical frequency so that $\mathcal{A}_{ \pm}\left(\vec{r}_{\perp} ; \omega\right) \equiv E_{ \pm}\left(\vec{r}_{\perp} ; \nu\right)$, with $\nu=\omega-\Omega$, is different from zero only in the close vicinity of $\Omega$. Thus, in the time domain, 
the circularly polarized components of the optical field can be written as

$$
\mathcal{A}_{ \pm}\left(\vec{r}_{\perp} ; t\right)=E_{ \pm}\left(\vec{r}_{\perp} ; t\right) e^{-i \Omega t}+c . c .
$$

where $E_{ \pm}\left(\vec{r}_{\perp} ; t\right)$ are the SVAs of the circularly polarized components of the optical field, which verify that $\left|\partial_{t} E_{ \pm}\right| \ll \Omega\left|E_{ \pm}\right|$.

For frequencies $\omega \simeq \Omega$, we approximate

$$
\begin{aligned}
\frac{\omega^{2}}{c^{2}} & {\left[1+\chi_{e}\left(\vec{r}_{\perp} ; \omega\right)\right]-q^{2} } \\
\approx & 2 \frac{\Omega}{c} n_{e} \frac{n_{g}}{c} \nu+2\left(\frac{\Omega}{c}\right)^{2} n_{e}\left[\Delta n\left(\vec{r}_{\perp} ; \Omega\right)+i \alpha_{i}(\Omega)\right] \\
& -2 i \frac{\Omega}{c} n_{e} \operatorname{Im} q(\Omega)
\end{aligned}
$$

where $n_{e} \equiv n_{e}(\Omega)$ is the effective index at the cavity frequency and $n_{g} \equiv\left|(d / d \omega)\left(\omega n_{e}-c \operatorname{Re} q\right)\right|_{\omega=\Omega}$ is the corresponding group refractive index. By neglecting the frequency dependence of $\delta \chi_{e}$ and $\delta q$ in (22) and transforming it to the time domain $\left(-i \nu \rightarrow \partial_{t}\right)$, we have that

$$
\begin{aligned}
& 2 i \frac{\Omega}{c} n_{e} \frac{n_{g}}{c} \partial_{t} E_{ \pm}\left(\vec{r}_{\perp} ; t\right) \\
&+\left\{2\left(\frac{\Omega}{c}\right)^{2} n_{e}\left[\Delta n\left(\vec{r}_{\perp} ; \Omega\right)+i \alpha_{i}(\Omega)\right]\right. \\
&\left.\quad-2 i \frac{\Omega}{c} n_{e} \operatorname{Im} q(\Omega)\right\} E_{ \pm}\left(\vec{r}_{\perp} ; t\right) \\
&+ {\left[\frac{\Omega^{2}}{c^{2}} \delta \chi_{e}\left(\vec{r}_{\perp} ; \Omega\right)-\delta q^{2}(\Omega)\right] E_{\mp}\left(\vec{r}_{\perp} ; t\right) } \\
&=-\Gamma \int_{-\infty}^{\infty} \frac{d \nu}{2 \pi} e^{-i \nu t} \frac{(\Omega+\nu)^{2}}{c^{2}} \\
& \cdot \chi_{ \pm}\left(\Omega+\nu, \bar{N}_{+}, \bar{N}_{-}\right) E_{ \pm}\left(\vec{r}_{\perp} ; \nu\right) .
\end{aligned}
$$

The right-hand side of (27) does not allow for an exact integration. However, by expanding $\chi_{ \pm}$to the first order in $\nu$, integrating term by term and formally resuming the series, we have that the temporal evolution of the transverse-field distribution $E_{ \pm}\left(\vec{r}_{\perp} ; t\right)$ is determined by

$$
\begin{aligned}
\partial_{t} E_{ \pm}= & -\kappa E_{ \pm}+i \hat{\mathcal{L}} E_{ \pm}+i \frac{\Gamma}{2} \frac{c}{n_{g}} \frac{\Omega}{c n_{e}} \\
& \cdot \chi_{ \pm}\left(\Omega+i \frac{\partial_{t} E_{ \pm}}{E_{ \pm}}, \bar{N}_{+}, \bar{N}_{-}\right) E_{ \pm} \\
& -\left[\bar{\gamma}_{a}\left(\vec{r}_{\perp} ; \Omega\right)+i \bar{\gamma}_{p}\left(\vec{r}_{\perp} ; \Omega\right)\right] E_{\mp} .
\end{aligned}
$$

In the above equation, we have defined $\kappa=\left(c / n_{g}\right)$ $\left[(\Omega / c) \bar{\alpha}_{i}(\Omega)-\operatorname{Im} q(\Omega)\right]$ as the total cavity loss rate, and

$$
\begin{aligned}
& \gamma_{a}\left(\vec{r}_{\perp} ; \Omega\right)=\frac{\Omega}{2 n_{e} n_{g}} \operatorname{Im}\left[\frac{\Omega^{2}}{c^{2}} \delta \chi_{e}\left(\vec{r}_{\perp} ; \Omega\right)-\delta q^{2}(\Omega)\right] \\
& \gamma_{p}\left(\vec{r}_{\perp} ; \Omega\right)=-\frac{\Omega}{2 n_{e} n_{g}} \operatorname{Re}\left[\frac{\Omega^{2}}{c^{2}} \delta \chi_{e}\left(\vec{r}_{\perp} ; \Omega\right)-\delta q^{2}(\Omega)\right]
\end{aligned}
$$

which represent the effective dichroism and birefringence in the cavity. Anisotropies have two different contributions: $\delta \chi_{e}$, which represents the anisotropies that arise from the passive material filling the cavity, and $\delta q^{2}$, which arises from the Bragg mirrors. For simplicity, we consider that $\gamma_{a}$ and $\gamma_{p}$ are constant, independent of both position and frequency.

The waveguide operator in (28) reads

$$
\hat{\mathcal{L}} E_{ \pm}=\frac{c^{2}}{2 \Omega n_{e} n_{g}}\left[\nabla_{\perp}^{2}+\left(\frac{\Omega}{c}\right)^{2} 2 n_{e} \Delta n\left(\vec{r}_{\perp} ; \Omega\right)\right] E_{ \pm}
$$

since we have assumed weak guidance, i.e., $\Delta n\left(\vec{r}_{\perp} ; \Omega\right) \ll n_{e}$. It is worth remarking that $\hat{\mathcal{L}}$ does not incorporate the carrier-induced refractive index, which is included separately through the real part of the susceptibility. However, all other guiding mechanisms and, in particular, thermal effects due to carrier injection are, indeed, included in $\hat{\mathcal{L}}$ because both the cavity frequency $\Omega$ and the excess refractive index distribution $\Delta n\left(\vec{r}_{\perp} ; \Omega\right)$ are sensitive to the injected current due to device self-heating. The eigenfunctions of $\hat{\mathcal{L}}$ are thus the cavity modes corresponding to the effective waveguide, and if this guide is strong enough, the interaction with the carriers will not distort them too strongly. It is worth remarking that with our definition of $\hat{\mathcal{L}}$, the cavity modes and modal frequencies are polarization independent. The linear cavity anisotropies are described through $\gamma_{a}$ and $\gamma_{p}$.

Finally, in (28), two points are worth remarking. In the first place, the optical frequency $\Omega$ is selected by the cavity through (24). In the second place, a correction to the optical frequency $\Omega$ appears through the "instantaneous frequency" $i \partial_{t} E_{ \pm} / E_{ \pm}$in $\chi_{ \pm}\left(\Omega+i \partial_{t} E_{ \pm} / E_{ \pm}, \bar{N}_{+}, \bar{N}_{-}\right)$. Such a contribution takes into account the changes in the susceptibility due to the frequency pulling or pushing due to nonlinearities and it also describes the variations in susceptibility experienced through frequency chirping during transients. But, more important, it also determines that the carrier-induced gain and refractive index experienced by different transverse modes are different due to their different modal frequencies.

\section{B. Material Model}

As already discussed, due to the quantum-mechanical selection rules that apply to optical transitions in the QW the interaction, with the active material is diagonal in the basis of circularly polarized states. It is then natural to split the total carrier density into spin-up and spin-down carrier densities. Each of them interacts with only one of the two circularly polarized components of the optical field, but scattering processes that reverse the spins of the carriers couple the two densities. We describe this coupling by means of an effective spin-flip rate $\gamma_{J}$ that phenomenologically describes the equalization of the densities of spin-up and spin-down carriers [2], [36]. The evolution of each of the spin-resolved densities can be found from the density matrix formalism [38] applied to semiconductor systems, which leads to [9], [24]

$$
\begin{aligned}
\partial_{t} \bar{N}_{ \pm}= & \frac{J\left(\vec{r}_{\perp} ; t\right)}{2 e W}-R_{s p}\left(\bar{N}_{ \pm}\right)+\mathcal{D} \nabla_{\perp}^{2} \bar{N}_{ \pm} \\
& \mp \gamma_{J}\left(\bar{N}_{+}-\bar{N}_{-}\right)+\frac{\Gamma}{W} \frac{2 \varepsilon_{0}}{\hbar} \int_{0}^{L} d z|f(z)|^{2} \\
& \cdot \operatorname{Im}\left[\chi_{ \pm}\left(\Omega+i \frac{\partial_{t} E_{ \pm}}{E_{ \pm}}, N_{+}, N_{-}\right)\right]\left|E_{ \pm}\right|^{2}
\end{aligned}
$$


where

$e$

$\mathcal{D}$

$R_{s p}\left(\bar{N}_{ \pm}\right)$

$\gamma_{J}\left(\bar{N}_{+}-\bar{N}_{-}\right)$

absolute value of the electron charge;

in-plane ambipolar diffusion coefficient;

$=A \bar{N}_{ \pm}+B \bar{N}_{ \pm}^{2}-$ total spontaneous recombination of carriers (we neglect Auger recombination);

all spin-flip processes that tend to equalize the two carrier densities with opposite spin.

Finally, $J\left(\vec{r}_{\perp} ; t\right)$ denotes the distribution of the current flowing through the active region, which is assumed to be equally distributed among the two spin orientations. Thus, the total injected current is $I=\int d^{2} \vec{r}_{\perp} J\left(\vec{r}_{\perp} ; t\right)$.

Our VCSEL model is given by (28) and (32), which together determine the distribution of the SVA fields and carrier densities. However, it still has to be closed by providing a specification for the optical susceptibility components, $\chi_{ \pm}$, that describe the interaction of the optical field and the QW material: their imaginary parts describe the energy exchange (absorption or stimulated emission) between the circular components of the field and the medium, while their real parts describe the dispersive effect (refractive index change) accompanying such a process [39]. Therefore, once the susceptibility components have been specified, our model naturally includes the effects of spatial-hole burning that leads to a reduction in the modal gain due to a depletion of the carrier density distribution, but also through a change both in the position of the modal frequencies on the gain spectrum and in the modal profiles. For index-guided devices, this last effect can be usually neglected and it is enough to determine the imaginary parts of $\chi_{ \pm}$as a function of the frequency and the carrier densities.

Models for calculating the gain and refraction index spectra from the electronic structure of the semiconductor material have been developed, some neglecting many-body effects [37], [40]-[46] and some taking them into account [25]-[29], [47], [48]. These microscopic theories describe individual transitions by the occupation of the initial and final electronic states, and the material polarization by superposing the contributions from each transition. A dynamical description of the lasing process then requires dealing with plenty of two-level-like systems, coupled among them by carrier scattering processes and by the optical field. In this way, all physical mechanisms in the material are accounted for, but the complexity of such a description is so high that it requires intensive numerical computation even without considering spatio-temporal dynamics.

In order to reduce the computational cost and to gain physical insight, it is convenient to use simpler descriptions for the optical susceptibility of semiconductor media. One possibility is to use a semi-analytical approximation for the optical gain (see, for instance, [49] and references therein) and then determine the refractive index by Kramers-Kronig relations. Another possibility is to use an analytical approximation to the full optical susceptibility [30]-[32], which although less accurate, still captures the essential features of the gain and index spectra. For this reason, we consider an analytical approximation to the optical susceptibility of the QW, equivalent to that given in [30], but for the circular components of the optical field. We proceed along the lines given in [30], and we consider that only one conduction and one (heavy-hole) valence band, both parabolic and degenerated for the two spin orientations, contribute to the gain [see Fig. 1]. This situation is appropriate for describing thin, strained QW where the light-hole band has substantially higher energy than the heavy-hole band, so it is not optically active until quite high carrier densities. By assuming charge neutrality and that the spin relaxation rate for the holes is very large [36], the hole density can be eliminated from the VCSEL dynamics. In this limit, we have that $N_{+}^{h} \approx N_{-}^{h}=\left(N_{+}+N_{-}\right) / 2$, where $N_{+}$ and $N_{ \pm}^{h}$ denote the densities of electrons and holes per spin orientation, respectively. Finally, assuming intraband quasi-equilibrium, the analytical expression for the optical susceptibility reads

$$
\begin{aligned}
\chi_{ \pm}(\Omega+\nu & \left.N_{+}, N_{-}\right)=-\chi_{0}\left[\ln \left(1-\frac{2 D_{ \pm}}{u+i}\right)\right. \\
+ & \left.\ln \left(1-\frac{D_{+}+D_{-}}{u+i}\right)-\ln \left(1-\frac{b}{u+i}\right)\right] .
\end{aligned}
$$

where the first term on the right-hand side represents the contribution of the electrons, the second that of the holes, and the third represents the susceptibility of the system when no carriers are excited. In the above equation, we have defined

$$
\begin{aligned}
\chi_{0} & =\frac{m|M|^{2}}{2 W \pi \varepsilon_{0} \hbar^{2}}, \quad b=\frac{\hbar k_{m}^{2}}{2 m \gamma}, \quad D_{ \pm}=\frac{\pi W \hbar}{m \gamma} \bar{N}_{ \pm} \equiv \frac{\bar{N}_{ \pm}}{N_{t}} \\
u & =\Delta+\frac{\nu}{\gamma}+\sigma\left(D_{-}+D_{+}\right)^{1 / 3}
\end{aligned}
$$

where

$m \quad$ reduced mass of the electron-hole pair;

$N_{t} \quad$ (total) transparency carrier density;

$|M|^{2}$ oscillator strength of the transition, assumed to be constant over the whole band;

$\gamma \quad$ width of the transition, assumed to be constant over the whole band.

The frequency dependence is incorporated through $u$, in which $\Delta=\left(\Omega-\omega_{g}\right) / \gamma$ measures the normalized detuning of the longitudinal mode resonance with respect to the nominal bandgap, and $\sigma\left(D_{-}+D_{+}\right)^{1 / 3}$ phenomenologically describes bandgap renormalization due to Coulomb interaction between electrons and holes, $\sigma$ being the bandgap renormalization parameter.

As discussed in detail in [30], the optical susceptibility given by (33) provides a good qualitative description of the characteristics of both gain and refractive index spectra, including bandfilling effects (i.e., the blueshift of the gain peak relative to the bandedge as the carrier density is increased) and the nonlinear dependence of the gain and index spectra on the carrier density. By using this approximation for $\chi_{ \pm}$in (28) and (32), the spatio-temporal description of the system incorporates the frequency dependence of both the gain and refractive index in a simple, although efficient and qualitatively accurate way. Anyway, it should be stressed once again that other approximations for the susceptibility components could be used. In particular, when one wishes to analyze in detail the behavior of a particular device, computational complexity arises because of the need for a realistic and accurate modeling of the gain and index spectra for the device under analysis. 


\section{Dimensionless Model}

As a final step, for the sake of clarity and numerical purposes, it is convenient to use a dimensionless version of the VCSEL model. To this end, we work with the carrier densities normalized to the transparency carrier density, $D_{ \pm}=\bar{N}_{ \pm} / N_{t}$, and we scale the fields as

$$
\begin{aligned}
& A_{ \pm}\left(\vec{r}_{\perp} ; t\right) \\
& \quad=\left[\frac{2 \varepsilon_{0} n_{g} n_{e}}{\hbar \Omega} \frac{\Gamma}{W N_{t}} \int_{0}^{L} d z|f(z)|^{2}\right]^{1 / 2} E_{ \pm}\left(\vec{r}_{\perp} ; t\right) .
\end{aligned}
$$

We also consider that the shape of the transverse current density distribution is fixed by the structure of the device, so that $J\left(\vec{r}_{\perp} ; t\right)=e W N_{t} C\left(\vec{r}_{\perp}\right) \mu(t)$, where $C\left(\vec{r}_{\perp}\right)$ is the current shape and $\mu(t)$ its time dependence; hence, the total injected current reads

$$
I(t)=\mu(t) e N_{t} W \iint_{-\infty}^{\infty} C\left(\vec{r}_{\perp}\right) d^{2} \vec{r}_{\perp} .
$$

Then, the final form for the model is

$$
\begin{aligned}
& \partial_{t} A_{ \pm}\left(\vec{r}_{\perp} ; t\right) \\
& =-\kappa A_{ \pm}+i \hat{\mathcal{L}} A_{ \pm}+i \frac{a \Gamma}{2} \chi_{ \pm}\left(\Omega+i \frac{\partial_{t} A_{ \pm}}{A_{ \pm}}, D_{+}, D_{-}\right) A_{ \pm} \\
& \quad-\left(\gamma_{a}+i \gamma_{p}\right) A_{\mp}+\sqrt{\beta D_{ \pm}} \xi_{ \pm}\left(\vec{r}_{\perp} ; t\right) \\
& \partial_{t} D_{ \pm}\left(\vec{r}_{\perp} ; t\right) \\
& =\frac{\mu(t)}{2} C\left(\vec{r}_{\perp}\right)-A D_{ \pm}-\left(B N_{t}\right) D_{ \pm}^{2} \mp \gamma_{j}\left(D_{+}-D_{-}\right) \\
& \quad+\mathcal{D} \nabla_{\perp}^{2} D_{ \pm}+a \operatorname{Im} \chi_{ \pm}\left(\Omega+i \frac{\partial_{t} A_{ \pm}}{A_{ \pm}}, D_{+}, D_{-}\right)\left|A_{ \pm}\right|^{2}
\end{aligned}
$$

where $a \equiv \Omega /\left(n_{e} n_{g}\right)$, and the susceptibility is that given in (33). In addition, we have phenomenologically added stochastic Langevin terms with zero mean $\left(\left\langle\xi_{ \pm}(x, y ; t)\right\rangle=0\right)$ and uncorrelated in both space, time, and polarization $\left(\left\langle\xi_{i}^{*}(x, y ; t) \xi_{j}\left(x^{\prime}, y^{\prime} ; t^{\prime}\right)\right\rangle=2 \delta_{i, j} \delta\left(x-x^{\prime}\right) \delta\left(y-y^{\prime}\right) \delta\left(t-t^{\prime}\right)\right.$ with $i, j=+,-)$ to the equation for each electric field in order to model spontaneous emission processes [8].

For the sake of simplicity, we assume that the lateral current distribution at the active layer is given in terms of explicit functional forms of $C(r)$. We approximate this function by a supergaussian distribution in the case of bottom-emitting devices, while a ring-shaped current distribution is taken for top-emitting devices. In the same way, the radial dependence of the excess refractive index $\Delta n(r)$ that arises from the TL effect is assumed to be parabolic. Although the electrical and thermal models have not been yet implemented, they may be included in our optical model in order to self-consistently determine the distribution in current density, temperature and optical field. A summary of the meaning and numerical values of the device and material parameters can be found in Table I.

\section{THRESHOLD ANALYSIS}

A basic step in the characterization and modeling of VCSELs is to determine their threshold properties. In particular,
TABLE I

DEVICE AND MATERIAL PARAMETERS

\begin{tabular}{ccrc} 
Symbol & Meaning & Value & Dimensions \\
\hline \hline$a \chi_{0}$ & effective gain constant & $1.3 \cdot 10^{4}$ & $\mathrm{~ns}^{-1}$ \\
$\Gamma$ & longitudinal confinement factor & 0.045 & - \\
$\gamma$ & polarization decay rate & 20 & $\mathrm{ps}^{-1}$ \\
$n_{\mathrm{e}}$ & background refractive index & 3.3 & - \\
$n_{g}$ & group refractive index & 3.5 & - \\
$\lambda$ & free-space wavelength & 0.85 & $\mu \mathrm{m}$ \\
$\sigma$ & bandgap shrinkage & 0.2 & - \\
$b$ & empty band contribution to $\chi$ & $10^{4}$ & - \\
$\kappa$ & cavity losses & 300 & $\mathrm{~ns}^{-1}$ \\
$\gamma_{a}$ & linear dichroism & 0.5 & $\mathrm{~ns}^{-1}$ \\
$\gamma_{p}$ & linear birefringence & 30 & $\mathrm{~ns}^{-1}$ \\
$A$ & non-radiative recombination rate & 1.0 & $\mathrm{~ns}^{-1}$ \\
$B$ & bimolecular recombination rate & $10^{-10}$ & $\mathrm{~cm}^{3} \mathrm{~s}^{-1}$ \\
$N_{t}$ & transparent carrier density & $10^{18}$ & $\mathrm{~cm}^{-3}$ \\
$\gamma_{J}$ & spin flip rate & 50 & $\mathrm{~ns}^{-1}$ \\
$\mathcal{D}$ & bimolecular diffusion & 0.4 & $\mu \mathrm{m}^{2} \mathrm{~ns}^{-1}$ \\
\hline
\end{tabular}

the difference in threshold currents for the different transverse modes provides a rough estimate of the modes that can be excited for a given current, although above threshold the excitation of higher order transverse modes is favored because of spatial hole burning. In addition, the modal profiles and frequencies can be modified, especially for very weak guiding. One of the primary effects of TL is to modify the threshold characteristics of the VCSEL by changing both the threshold current and the mode selected at threshold.

The threshold current and the transverse mode selection for a given VCSEL can be determined in a simple way by analyzing the linear stability of the "off" state, i.e., $A_{ \pm}=0$. We apply to the "off" state a small perturbation $\delta A_{ \pm}\left(\vec{r}_{\perp}, t\right)$ in such a way that stimulated emission can be neglected in determining the carrier densities. Hence, from (37), we have that the carrier densities in each spin orientation are equal $-D_{+}=D_{-}=$ $D_{s}\left(\vec{r}_{\perp}\right)$ - since electrons with opposite spin orientations are, on average, equally injected, and given by

$$
0=\frac{\mu}{2} C\left(\vec{r}_{\perp}\right)-A D_{s}-\left(B N_{t}\right) D_{s}^{2}+\mathcal{D} \nabla_{\perp}^{2} D_{s}
$$

The solutions of (38) in turn determine the inhomogeneous distribution of the optical susceptibility, that in this case is the same for the two polarization components. The dynamics of $\delta A_{ \pm}\left(\vec{r}_{\perp} ; t\right)$ is given by

$$
\begin{aligned}
& \partial_{t} \delta A_{ \pm}=-\kappa \delta A_{ \pm}+i \hat{\mathcal{L}} \delta A_{ \pm}+ i \frac{a \Gamma}{2} \chi\left(\Omega+i \frac{\partial_{t} \delta A_{ \pm}}{\delta A_{ \pm}}, D_{s}\right) \\
& \cdot \delta A_{ \pm}-\left(\gamma_{a}+i \gamma_{p}\right) \delta A_{\mp} \cdot
\end{aligned}
$$

It is natural to expand the perturbation in modes of the waveguide operator $\hat{\mathcal{L}}$

$$
\delta A_{ \pm}\left(\vec{r}_{\perp} ; t\right)=\sum_{m l} \delta A_{m l} e^{ \pm i \Psi+\lambda_{m l} t} \Phi_{m l}\left(\vec{r}_{\perp}\right) e^{-i \omega_{m l} t}
$$


where $\delta A_{m l}$ stands for the perturbation's amplitude and $\lambda_{m l}$ is the perturbation's eigenvalue in each of these modes. Phaselocking among the two circularly polarized components at $\Psi=$ $0, \pi / 2$ provides linearly polarized light along the $\hat{x}$ and $\hat{y}$ axes, respectively. The cavity modes $\Phi_{m l}$ and the modal frequencies $\omega_{m l}$ are determined by the eigenvalue problem

$$
\left[\nabla_{\perp}^{2}+\left(\frac{\Omega}{c}\right)^{2} 2 n_{e} \Delta n_{t l}(r)\right] \Phi_{m l}=W_{m l} \Phi_{m l} .
$$

These linearly polarized modes $L P_{m l}$ are indexed with $m=$ $1,2, \ldots$ and $l=0, \pm 1, \ldots$. The mode profile has $(m-1)$ zeros in the radial direction, whereas $(2 l)$ zeros are in the angular direction. $L P_{10}$ is referred as the fundamental mode, $L P_{11}$ the first-order transverse mode, and the remaining as higher order transverse modes. An important property of $\Phi_{m l}$ in calculations is that represents a complete set of orthogonal eigenfunctions. It is worth recalling that these cavity modes and modal frequencies are polarization independent, and that the cavity anisotropies that may favor one linearly polarized state over the orthogonal one have been included through $\gamma_{a}$ and $\gamma_{p}$ [see (47) below].

As we have already commented, the refractive index distribution is approximated by a truncated parabolic profile

$$
\Delta n_{t l}(R)= \begin{cases}\Delta n_{t l}\left[1-R^{2}\right], & \text { if } R<1 \\ 0, & \text { if } R \geq 1\end{cases}
$$

with $R \equiv 2 r / \phi_{g}, \phi_{g}$ being the TL diameter. The modes of such a waveguide can be analytically expressed as a series expansion when $R<1$ and read [50]

$$
\Phi_{m l}(R, \theta)=e^{i l \theta} \begin{cases}\frac{\sum_{n=0}^{\infty} a_{n} R^{n+l}}{\sum_{n=0}^{\infty} a_{n}}, & \text { if } R<1 \\ \frac{K_{l}\left(W_{m l} R\right)}{K_{l}\left(W_{m l}\right)}, & \text { if } R \geq 1\end{cases}
$$

where $K_{l}$ is a second kind Bessel function of order $l$ and the coefficients $a_{n}$ in (43) are given by the recursive relations

$$
\begin{aligned}
a_{0}= & \text { arbitrary } \\
a_{2}= & -\frac{\left(V^{2}-W_{m l}^{2}\right) a_{0}}{4(l+1)} \\
a_{2 n}= & \frac{1}{4 n(n+l)}\left(V^{2} a_{2 n-4}-\left(V^{2}-W_{m l}^{2}\right) a_{2 n-2}\right), \\
& \quad \text { if } n \geq 2 \\
a_{2 n+1}= & 0
\end{aligned}
$$

$V=(2 \pi / \lambda)\left(\phi_{g} / 2\right) \sqrt{2 n_{e} \Delta n_{t l}}$ is the waveguide parameter. The guided modes are those verifying $0 \leq W_{m l} \leq V$, and their propagation constants $\beta_{m l}^{2}=\left(k_{0} n_{e}\right)^{2}+\left(2 W_{m l} / \phi_{g}\right)^{2}$ are obtained by imposing the boundary conditions at $R=1$, which yield a transcendental equation for $W_{m l}$

$$
W_{m l} \frac{K_{l+1}\left(W_{m l}\right)}{K_{l}\left(W_{m l}\right)}=-\frac{\sum_{n=0}^{\infty} n a_{n}}{\sum_{n=0}^{\infty} a_{n}} .
$$

From (31) and (36), the modal frequencies, referred to $\Omega$,can be written

$$
\omega_{m l}=-\frac{2 c^{2}}{\Omega n_{e} n_{g}}\left(\frac{W_{m l}}{\phi_{g}}\right)^{2} .
$$

Inasmuch as $\omega_{m l}<0$, it describes the redshift of the modes due to the thermal lensing with respect to the flat index distribution. The greater the TL strength, the larger this redshift, which also increases as the mode order $\mathcal{O}=(2 m+l-1)$ decreases.

Upon substituting (40) into (39) and projecting onto a mode $L P_{m l}$, the perturbation's eigenvalue $\lambda_{m l}$ is given by the solution of the implicit equation

$$
\begin{aligned}
& \lambda_{m l}=-\kappa \mp\left(\gamma_{a}+i \gamma_{p}\right) \\
& +i \frac{a \Gamma}{2} \frac{\iint \chi\left(\Omega+\omega_{m l}+i \lambda_{m l}, D_{s}(r ; \mu)\right)\left|\Phi_{m l}\right|^{2} d^{2} \vec{r}}{\iint\left|\Phi_{m l}\right|^{2} d^{2} \vec{r}}
\end{aligned}
$$

where the sign $-(+)$ corresponds to $\hat{x}(\hat{y})$ linearly polarized light. The perturbation's growth rate is $\operatorname{Re} \lambda_{m l}$, while it oscillates at a frequency shifted by $\operatorname{Im} \lambda_{m l}$ with respect to $\Omega+\omega_{m l}$. The integral term on the right-hand side of (47) describes the modal gain (real part) and the nonlinear frequency shift (imaginary part) of the transverse mode under consideration, taking into account any possible frequency pulling or pushing of the modal frequencies due to the carrier induced refractive index change.

Therefore, the threshold current for every linearly polarized transverse mode $L P_{m l}, \mu_{t h}(m, l, \pm ; \Delta)$ is found from the condition $\operatorname{Re} \lambda_{m l}=0$. Threshold currents for the two linearly polarized solutions $( \pm)$ are slightly different in the presence of linear anisotropies; hence, we define the threshold for a given transverse mode as

$$
\mu_{t h}(m, l ; \Delta) \equiv \min _{ \pm}\left\{\mu_{t h}(m, l, \pm ; \Delta)\right\}
$$

and the absolute laser threshold is thus determined by

$$
\mu_{t h}(\Delta) \equiv \min _{m, l}\left\{\mu_{t h}(m, l ; \Delta)\right\} .
$$

It is worth remarking that, given the carrier density distribution associated with the current injection in (38), the threshold current of transverse modes is determined jointly by the modal frequencies that establish the material gain, and the overlap of the modal profile with the carrier distribution. These effects are, in turn, dependent on both the relative detuning and the thermal lensing strength. In the next two subsections, we discuss the 


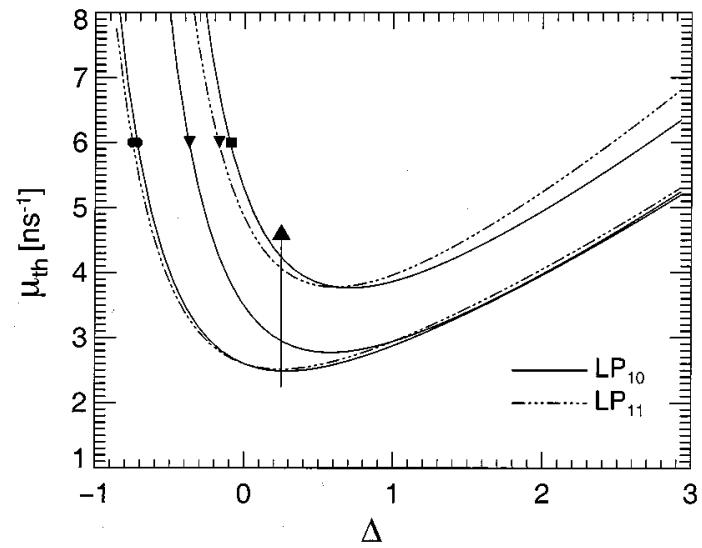

Fig. 2. Threshold curves for the fundamental (solid lines) and first-order transverse modes (dash lines) as function of the normalized detuning. The thermal lensing strength is $\Delta n_{t l}=10^{-2}(\bullet), \Delta n_{t l}=10^{-3}(\boldsymbol{\nabla}), \Delta n_{t l}=$ $5 \cdot 10^{-4}(\boldsymbol{\square})$.

threshold behavior of bottom and top emitter VCSELs corresponding to different shapes of the injected current.

\section{A. Bottom-Emitting VCSEL}

The current distribution across the active region of bottom-emitting VCSELs is quite homogeneous due to their circular $\mathrm{p}^{+}$contact and the typical high doping levels of the n-substrate [18], although current crowding at the aperture edges is observed when the VCSEL diameter is large. As we have already commented, we consider that for this device structure the current distribution at the active layer is super-Gaussian, $C(r)=\exp \left[-\left(2 r / \phi_{c}\right)^{6}\right]$, with $\phi_{c}$ being the diameter of the active region. The total injected current is then $I(t)=\mu(t)(\pi / 12) \Gamma(1 / 3) e N_{t} W \phi_{c}^{2}$, and the exponential tails of the supergaussian function take into account the current spreading effect [51]. We consider a device with an active region diameter $\phi_{c}=15 \mu \mathrm{m}$, and a larger diameter of the thermal lens, $\phi_{g}=18 \mu \mathrm{m}$, in order to mimic heat diffusion across the cavity axis.

In Fig. 2, we represent the threshold curves obtained from (48) for the fundamental (solid lines) and first-order (dashed-dotted lines) transverse modes as function of the normalized detuning $\Delta$. We consider different values of the TL refractive index strength $\Delta n_{t l}=10^{-2}, \Delta n_{t l}=10^{-3}$, and $\Delta n_{t l}=5 \cdot 10^{-4}$. For a fixed $\Delta n_{t l}$, the threshold curves for the different modes as a function of the detuning display a minimum when the modal frequency aligns with the gain peak. The position of such a minimum depends mainly on the thermal waveguide, but also on the coupling with the carrier density through bandfilling and bandgap shrinkage as included in $\chi$. The curves are asymmetric around the minimum threshold with a smoother increase toward the blue side of the gain spectrum as a result of a higher differential gain. For small $\Delta n_{t l}$, which corresponds to an on-axis temperature excess of about $1 \mathrm{~K}$, we observe that besides the global increase of the threshold current, the threshold for the first-order transverse mode is very large (out of scale in Fig. 2). For moderate $\Delta n_{t l}$, the threshold of the fundamental mode is sensibly smaller than the first-order transverse mode, so the laser displays fundamental transverse mode operation for moderate currents above threshold. We observe that the threshold discrimination of the first-order transverse mode is more noticeable when the cavity resonance is located on the blue side of the gain curve, although in this case the threshold current increases. For large $\Delta n_{t l}$, we observe that the threshold differences between the two modes are very small over the whole range of detunings, thus indicating a strong tendency toward multimode emission. An interesting aspect is that, when the VCSEL operates on the red side of the gain spectrum, the first-order transverse mode has a lower threshold than the fundamental one.

This general scenario can be interpreted from (47) as the interplay of two separate aspects. On one hand, the TL waveguide establishes the modal profiles, $\Phi_{m l}$ and frequencies $\omega_{m l}$. The latter alone would define the modal gain if the active region were of infinite extent and injected homogeneously. Then, the threshold curves for the different modes would follow the material gain spectrum and one would, therefore, expect that the threshold mode would be the one whose frequency is the closest to the gain peak. However, due to the finite extent of the carrier density distribution, a geometrical correction sets in that accounts for the overlap of the carrier density and the mode profile. This effect is usually described by means of a lateral confinement factor that corresponds to the fraction of the modal power contained in the nucleus of the waveguide. In our case, however, the carrier distribution is inhomogeneous; thus, we define a lateral confinement factor through

$$
\Gamma_{m l} \equiv \frac{\iint\left|\Phi_{m l}\right|^{2} \mathcal{S}\left(\vec{r}_{\perp}\right) d^{2} \vec{r}}{\iint\left|\Phi_{m l}\right|^{2} d^{2} \vec{r}}
$$

where $\mathcal{S}\left(\vec{r}_{\perp}\right)$ is a normalized weight function, ranging from zero to one, that describes the shape of the active region. For simplicity, we take $\mathcal{S}(r) \equiv D_{s}(r) / \max \left\{D_{s}(r)\right\}$ with $D_{s}(r)$ the steady-state carrier distribution given by (38). When the carrier density in the active region is approximated by a disc $\mathcal{S}(r)=\Theta\left(\phi_{c} / 2-r\right)$, our definition of the lateral confinement coincides with the fraction of modal power within the active region. With our choice for $\mathcal{S}$, the lateral confinement factor describes the degree of overlap of the modal profiles with the carrier distribution. Note that $\Gamma_{m l}$ does not depend either on the modal frequencies or on the cavity detuning. Hence, we are able to separate the geometrical contributions to the laser threshold from those arising from the frequency dependence of the gain curve.

The lateral confinement as obtained from (50) for the guided modes considered above is depicted in Fig. 3. As expected, we observe a fast decrease of the confinement factors as the TL strength decreases, suddenly dropping to zero when the mode is no longer confined by the TL waveguide. It is clear from Fig. 3 that for strong TL, the confinement factors for the fundamental and the first-order transverse modes become very similar and close to one. In such a case, the material gain differences arising from different modal frequencies may be large enough to overcompensate for the difference in confinement factors. Hence, the device can start to lase in the first-order transverse mode in spite of being homogeneously pumped. In order to improve the 


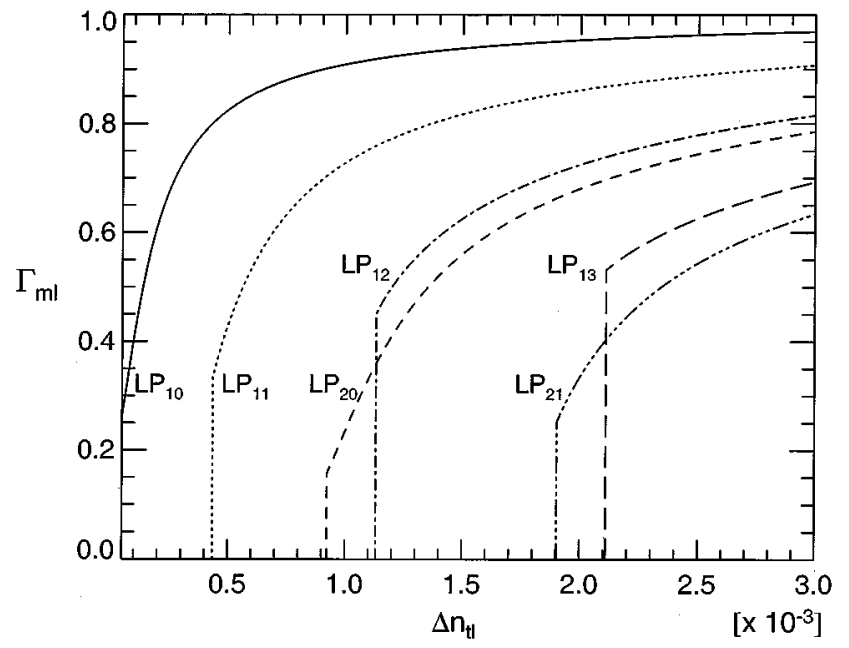

Fig. 3. Lateral confinement factor of the modes supported by the TL waveguide as function of the TL strength. The carrier distribution corresponds to the bottom-emitter VCSEL.

range of single-mode operation, it is desirable to work below the cut-off for the first-order transverse mode, which-for a fixed geometry - can be achieved by reducing the amount of TL through an increase of the device's lateral heat conductivity. For our particular configuration, the TL strength has to be moderate, and our analysis suggests that the on-axis excess temperature should be kept below $\sim 5 \mathrm{~K}$, since in this case, the confinement factor for the first-order transverse mode is already only $7 \%$ below that of the fundamental mode. However, it must be noted that the first-order transverse mode could start lasing well above threshold due to spatial-hole burning in the carrier density.

\section{B. Top-Emitting VCSEL}

Top-emitting VCSELs have a top contact of annular shape that leads to preferential injection in the outer edges of the active region both due to the ring contact and to current crowding at the aperture edges in the case of oxidized VCSELs. This strongly affects the overlap of the modal profiles with the carrier density, and thus the mode selection at threshold. In order to illustrate this effect, we take the radial dependence of the injected current as: $C(\rho)=e^{-\rho^{6}} e^{\rho^{2}}$, with $\rho=2 r / \phi_{c}$ in such a way that the total injected current in the device is $I(t) \approx 1.24 \mu(t) e W N_{t} \phi_{c}^{2}$. The variation in carrier density from the center to the carrier crowding radius is of the order of $\Delta N / N(r=0)=25 \%$ for the actual diffusion coefficient. For simplicity and an easier comparison with the bottom-emitting VCSEL, we assume that the thermal lensing profile is unaffected by the ring in the current distribution, although such an approximation is unrealistic in the case of small radial thermal conductivity and when a marked ring-shaped current distribution is considered.

The threshold curves of the transverse modes, for a moderate value of TL $\left(\Delta n_{t l}=5 \cdot 10^{-3}\right)$ are shown in Fig. 4. The fundamental transverse mode is unfavored, with respect the other modes, due to its poor overlap with the carrier density resulting from the ring-shaped current injection. For these specific operating conditions, the lowest threshold corresponds, over the

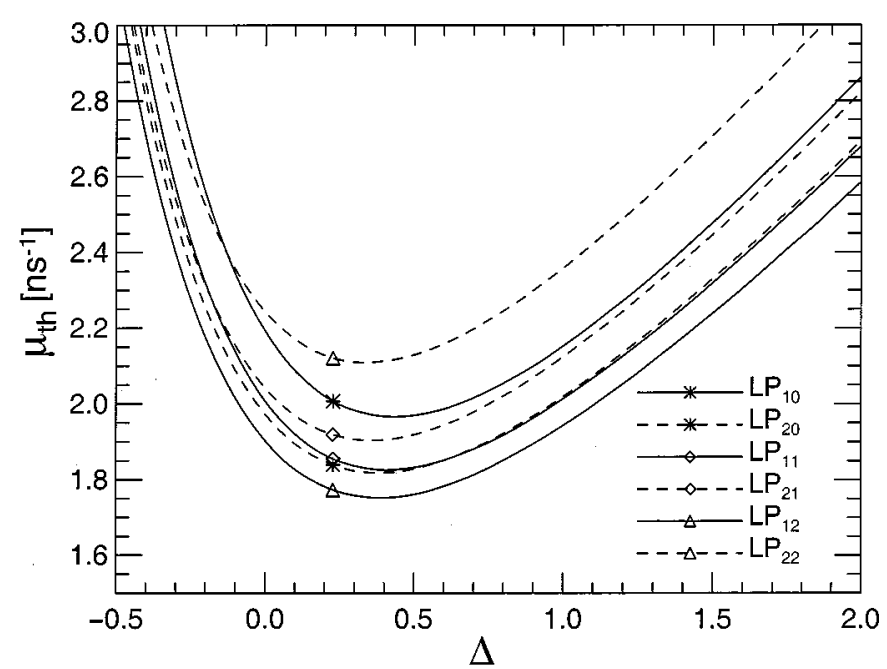

Fig. 4. Threshold curves for the successive transverse modes of the top-emitter VCSEL. The position of the symbols denote the detuning of operation and its corresponding threshold current. The thermal lensing strength is $\Delta n_{t l}=5$. $10^{-3}$.

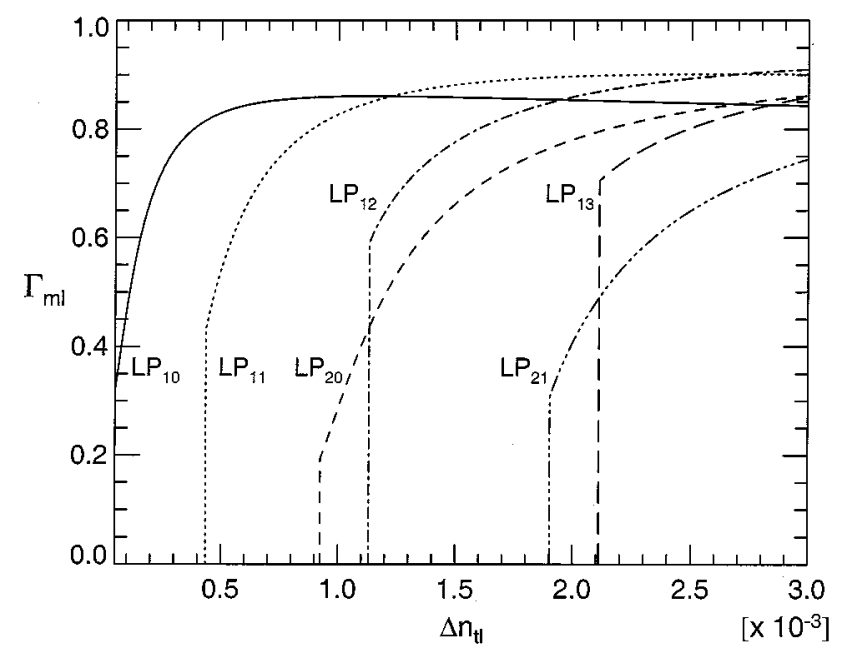

Fig. 5. Lateral confinement factor of the modes supported by the TL waveguide as function of the TL strength. The carrier distribution corresponds to the top-emitter VCSEL.

whole range of detunings, to the four lobed $L P_{12}$ mode. Again, it can be observed that the range of single-mode operation of the device can be improved by detuning the cavity resonance to the blue side of the gain peak. It must be noted, however, that in this case, the $L P_{12}$ mode is further away from the gain peak than modes of lower order, hence clearly showing the dominance of the geometrical effects over the material gain.

The lateral confinement factor defined in (50) is plotted in Fig. 5 versus the TL strength. As the TL strength is increased, we observe that different transverse modes are favored depending on the TL conditions. From this purely geometrical point of view, the fundamental mode $L P_{10}$ tends to dominate due to a better overlap with respect to the other modes for $\Delta n_{t l}<$ $1.2 \cdot 10^{-3}$. For $1.2 \cdot 10^{-3}<\Delta n_{t l}<2.7 \cdot 10^{-3}$, the first-order transverse mode $L P_{11}$ is favored, while for $\Delta n_{t l}>2.7 \cdot 10^{-3}$, the four-lobed $L P_{12}$ displays the maximum confinement factor. 

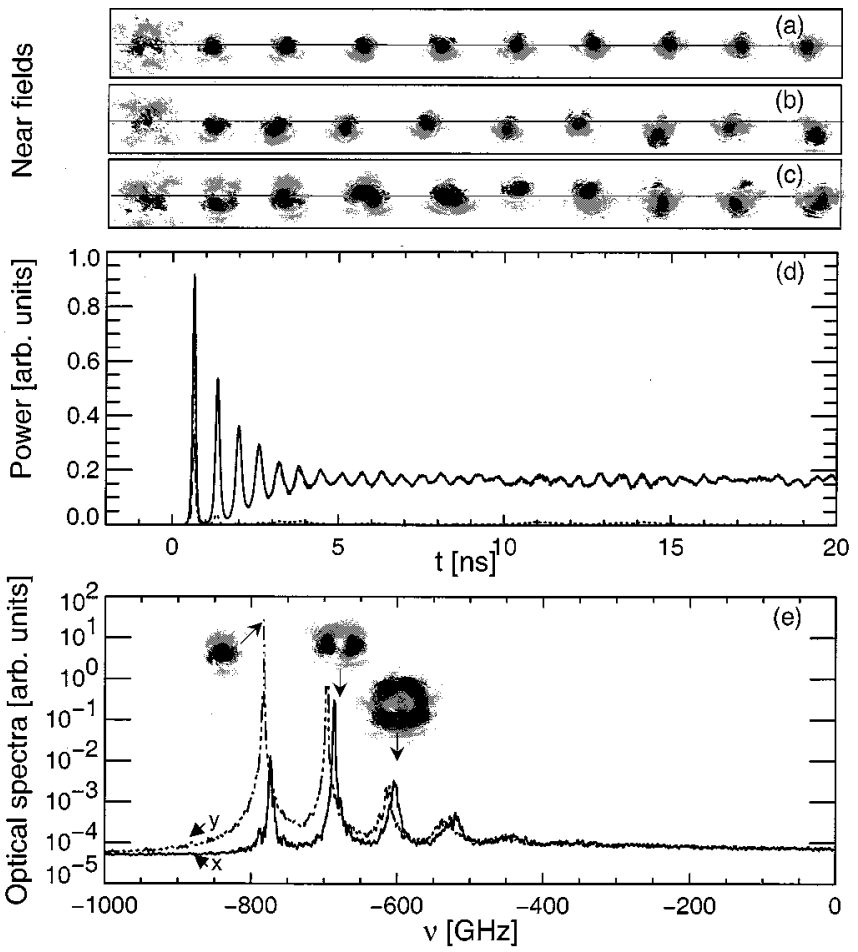

Fig. 6. Switch-on dynamics of the bottom emitter VCSEL. Evolution of the total intensity near-fields: (a) $\mu=1.05 \mu_{t h}$; (b) $\mu=1.10 \mu_{t h}$; and (c) $\mu=1.25 \mu_{t h}$. (d) Temporal evolution of the total intensity at $\mu=1.10 \mu_{t h}$. (e) Optical spectra of both linear polarizations under $\mathrm{CW}$ operation at $\mu=1.10 \mu_{t h}$.

\section{NUMERICAL RESULTS}

In order to give more specific evidences of the interplay of $\mathrm{TL}$ and the shape of the gain distribution, we perform numerical simulations of (33), (36), and (37). In a first instance, we discuss the switch-on dynamics of bottom- and top-emitting VCSELs when they operate close to threshold. In a second instance, we analyze the response to a short current pulse going from below to well above threshold. In particular, we analyze the spectral properties of these devices when the thermal lensing strength changes. Some guidelines about the numerical integration scheme can be found in the Appendix.

\section{A. Laser Switch-On}

In this section, we assume that the nominal detuning is $\Delta=$ 0.25 , chosen to achieve operation near the gain peak, and we take a moderate value for the TL strength: $\Delta n_{t l}=5 \cdot 10^{-3}$. In these conditions, the threshold analysis presented in the previous section shows that the mode with the lowest threshold is the fundamental one, with $\hat{y}$-polarization. In Fig. 6, we show the dynamics of the bottom-emitting VCSEL when it is biased close to threshold. The evolution of the total intensity near fields is shown in panels Fig. 6(a)-(c) for three different currents ranging from $\mu=1.05 \mu_{t h}$ up to $\mu=1.25 \mu_{t h}$. Near-field images are plotted with an inverted gray-scale scheme using maximum contrast. Therefore, comparison of the relative intensity between images is not possible. For the lower injection current, we obtain stable fundamental mode operation in a well-established polarization. When the current is slightly increased, the near field is still Gaussian, but its position changes from image to image.
Increasing further the injection current, we find that this last stage ignites the appearance of the first-order transverse mode as can be clearly seen in Fig. 6(c). As is commonly observed, the first-order transverse mode switches on in the orthogonal polarization of the lasing one. We note that this result is not a direct consequence of the threshold analysis presented in Section III, but a nonlinear competition between transverse and polarization degrees of freedom. The total intensity evolves accordingly to Fig. 6(d) when the current is $\mu=1.10 \mu_{t h}$. The VCSEL emits preferentially in the lower frequency polarization component $(\hat{y}$-LP), being selected by the actual value of the dichroism. The orthogonal component is considerably suppressed in $\mathrm{CW}$, although it appears during the transient following the switch-on. The optical spectrum of the dominant polarization, under $\mathrm{CW}$ operation, displays a dominant peak at the position of the fundamental transverse mode frequency. The orthogonal polarization component clearly exhibits the dominance of the first-order transverse mode. It is worth remarking that the current at which such a mode appears is smaller than that predicted by the threshold analysis due to the role of the spatial hole burning, i.e., the steady-state carrier distribution for this current is no longer super-Gaussian, but displays a hole at the center due to the increased stimulated recombination in this region.

In view of the above results, one might think to preferentially excite one of the transverse modes by proper selection of the current profile. To explore this possibility, we perform numerical simulations of the top-emitting VCSEL biased close to the threshold current. The current is switched on from slightly below threshold to above threshold $\mu=1.1 \mu_{t h}$. In Fig. 7(a), we represent the evolution of the total intensity accompanied by the near-field images at different stages. In the "off" state, the spontaneous emission near field displays a hole at the center as a result of the ring-shaped carrier distribution. As soon as the laser switches on, we observe that a transverse mode with four lobes is selected. Nevertheless, the orientation of the mode is not fixed and starts to rotate, alternating between odd and even $L P_{12}$ modes. The polarization-resolved optical spectra, computed under CW operation [see Fig. 7(b)] reveals that the device exhibits nearly single-mode operation with a predominant peak that corresponds to the four-lobed $L P_{12}$ mode. However, a daisy mode with six lobes is weakly excited ( $\sim 40 \mathrm{~dB}$ of side-mode suppression ratio). The four-lobed structure of the $L P_{12}$ mode burns a hole in the carrier distribution along the angular direction that induces the rotation of the mode, and this yields the weak excitation of the daisy mode. We also note reminiscent peaks, with much lower power, at the frequency positions of the fundamental and first-order transverse modes.

\section{B. Response to a Current Pulse}

In this second example, the cavity detuning is kept at $\Delta=$ 0.25 and the VCSEL is subject to an electrical excitation that consists in a current pulse of $1 \mathrm{~ns}$ in duration and $50 \mathrm{ps}$ of rise and fall times. The current is switched on at $t=0$ from $\mu_{b}=0.85 \mu_{t h}$ to well above threshold $\mu_{o n}=4 \mu_{t h}$. Under these conditions, the thermal profile in the VCSEL is kept constant during the current pulse since the typical time scales for the thermal response are of the order of $\tau_{t} \sim 1 \mu \mathrm{s}$. In addition, 

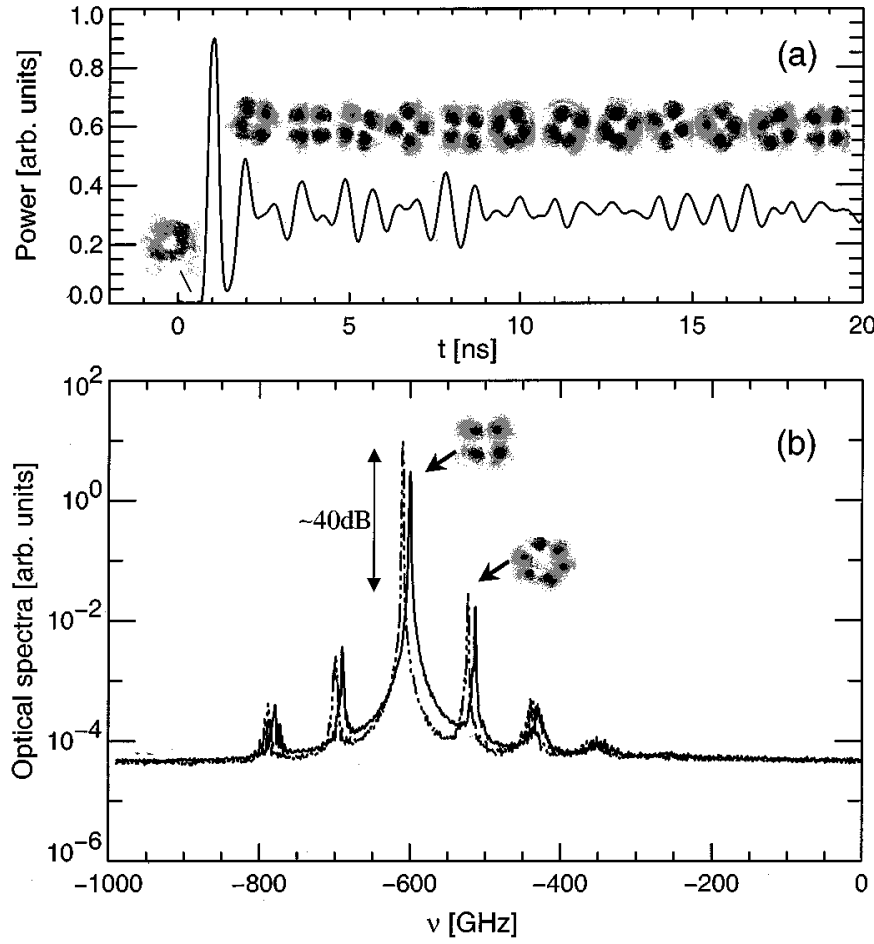

Fig. 7. Higher-order transverse mode selection for the top-emitter VCSEL. (a) Close-to-threshold temporal evolution of the total intensity at $\mu=1.10 \mu_{t h}$. (b) Corresponding optical spectra in both polarizations under $\mathrm{CW}$ operation. Near-field images are obtained using the maximum contrast of an inverted gray-scale scheme.

the effective value of the TL strength is determined by the operating bias current.

We consider in the first place the transient response of the bottom-emitter VCSEL for three different TL strengths: $\Delta n_{t l}=10^{-2}, \Delta n_{t l}=5 \cdot 10^{-3}$, and $\Delta n_{t l}=5 \cdot 10^{-4}$. In Fig. 8, we represent snapshots of the power distribution in the two linear components. When analyzing the spatio-temporal response to the current pulse, we observe that laser switches-on in the fundamental transverse mode followed by the successive excitation of higher-order transverse modes. The weaker the TL, the smaller the number of excited modes with much longer turn-on times. In Fig. 9, we show the polarization-resolved optical spectra corresponding to the previous dynamics. Both linear polarizations $\hat{x}$ LP and $\hat{y}$-LP are depicted by solid and dashed lines, respectively. We find that the frequency separation between successive transverse modes, which is approximately constant for a parabolic waveguide, depends drastically on the TL properties. We obtain a frequency separation between the fundamental and first-order transverse mode of 120, 80, and $54 \mathrm{GHz}$ (Fig. 9(a)-(c), respectively). For the first two cases, these frequency differences agree quite well with those predicted by $(46),\left(\omega_{11}-\omega_{10}\right) /(2 \pi)=118 \mathrm{GHz}$ and $83 \mathrm{GHz}$, respectively. However, this is not so in Fig. 9(c), where (46) predicts a frequency difference of $23 \mathrm{GHz}$. This is because the waveguide distortion caused by the carrier-induced refractive index strongly modifies the modal profiles and frequencies. In this case, the analysis performed in Section III is no longer valid, and alternative methods that take into account these effects are required for its calculation [52]. In addition, we

\section{(a)}

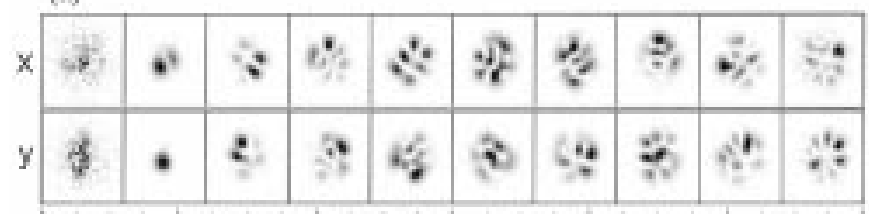

(b)

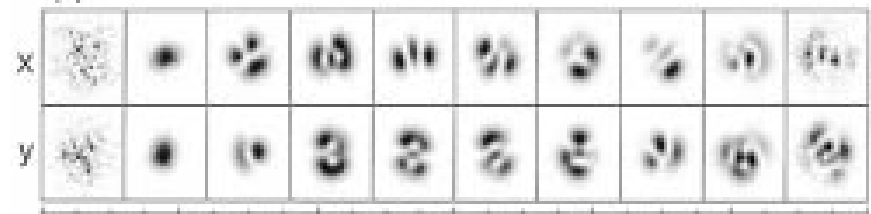

(c)

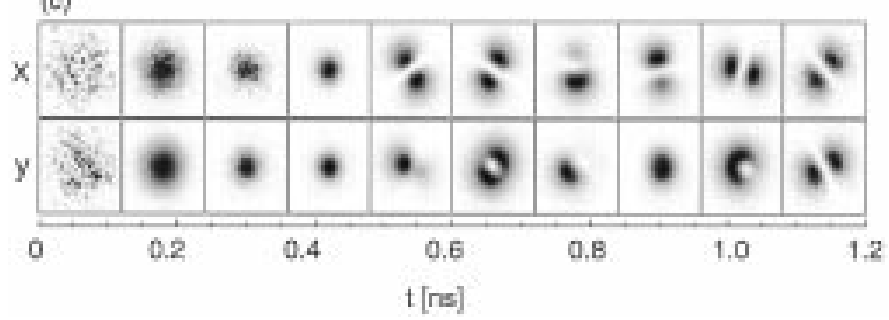

Fig. 8. Response to a current pulse of the bottom-emitter VCSEL, $\mu_{b}=0.85 \mu_{t h}$ and $\mu_{o n}=4 \mu_{t h}$. Snapshots of the near-field power distribution in $\hat{x}$ and $\hat{y}$ polarization components. Thermal lensing strength: (a) $\Delta n_{t l}=10^{-2}$; (b) $\Delta n_{t l}=5 \cdot 10^{-3}$; and (c) $\Delta n_{t l}=5 \cdot 10^{-4}$.
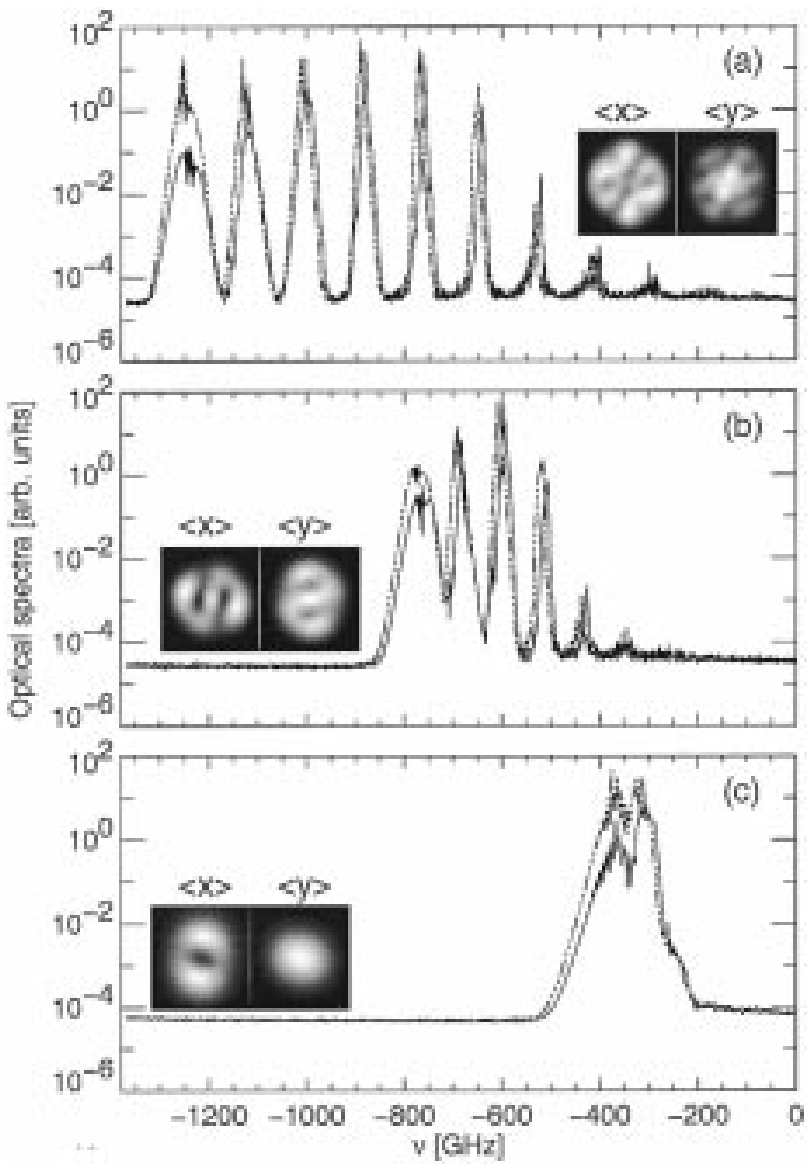

Fig. 9. Polarization resolved optical spectra of the bottom-emitter VCSEL, $\hat{x}$-LP (solid lines) and the $\hat{y}$-LP (dashed lines). The thermal lensing strengths correspond to those used in Fig. 8. Near-field images depict the time averaged power distribution in both linear polarization components. 

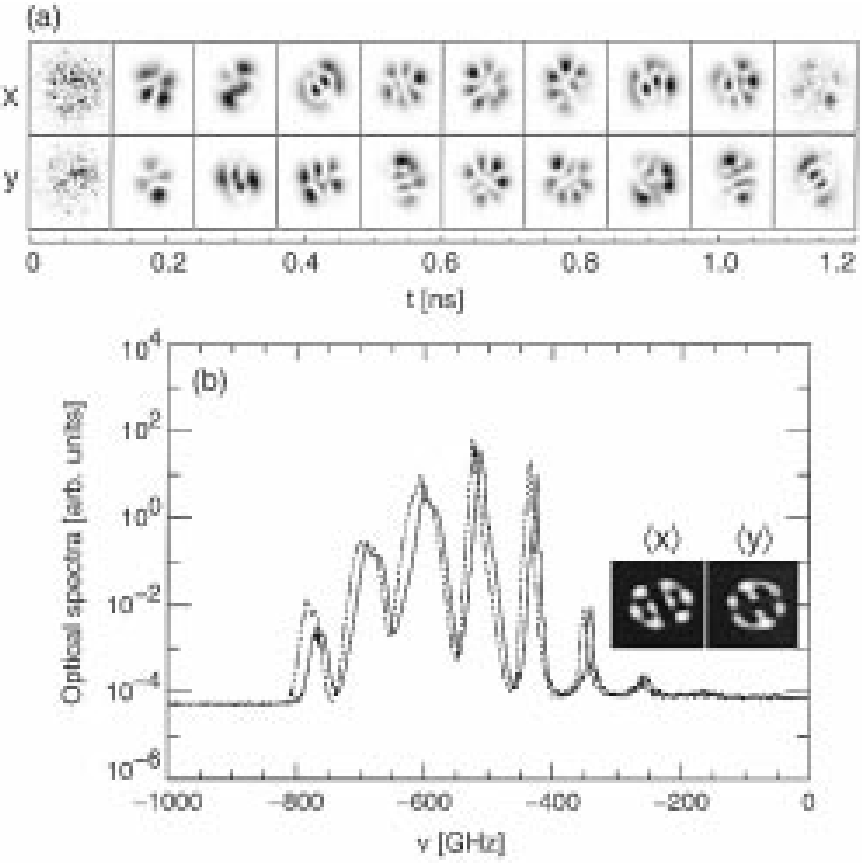

Fig. 10. Response to a current pulse of the top-emitter VCSEL, $\mu_{b}=0.85 \mu_{t h}$ and $\mu_{o n}=4 \mu_{t h}$. (a) Snapshots of the near-field power distribution in $\hat{x}$ and $\hat{y}$ polarization components. (b) Optical spectra and near-field images corresponding to the time-averaged intensity distribution in each linear polarization. The thermal lensing strength is $\Delta n_{t l}=5 \cdot 10^{-3}$.

can see that both polarizations are active during the transient regime displaying similar dynamics. Their spectra show a birefringence splitting of $10 \mathrm{GHz}$ that corresponds to $\gamma_{p} / \pi$. The near-field images in this figure depict the time-averaged power distribution in each linear polarization. As consequence of the number of modes excited by the current pulse, we go from complex near fields resulting from the superposition of several transverse modes Fig. 9(a) to simpler ones Fig. 9(c).

The spatio-temporal response of the top-emitting VCSEL to the same current pulse, for $\Delta=0.25$ and $\Delta n_{t l}=5 \cdot 10^{-3}$, is depicted in Fig. 10(a). In contrast with the bottom-emitter VCSEL, the laser onset is initiated in a higher order transverse mode. The corresponding optical spectra are shown in Fig. 10(b). First, we note that the high current pulse induces the excitation of several transverse modes, certainly more than in Fig. 7(b). We observe that the dominant peak in the optical spectrum now corresponds to the daisy mode, instead of the $L P_{12}$ mode, although this is the mode that is favored in CW operation as discussed before. The dominance of the daisy mode is confirmed by the time-averaged near-field power distributions, which display the preference for the emission in the daisy mode in both linear polarizations. Nevertheless, many other modes carry substantial power during this transient, during which the side-mode suppression ratio of the emission is strongly degraded. The reason is that, after the application of the current pulse and until the switch-on occurs, the carrier density increases well above its threshold value. This effect induces a blueshift of the gain peak that provides transient extra gain to all the modes, preferentially to higher order ones, which can then start to lase during a short period. Associated with this relatively large variation of carrier density, the carrier-induced refractive index also exhibits large variations that reflect themselves in the much broader peaks of the optical spectrum as compared with the case of $\mathrm{CW}$ operation. This effect is usually described by means of the linewidth enhancement factor, but in our model, the inclusion of the full susceptibility into the VCSEL dynamics already accounts for it.

\section{SUMMARY AND DISCUSSION}

We have developed an optical model for the large-signal dynamics of multimode VCSELs that incorporates both spatial and polarization degrees of freedom simultaneously. We have assumed that the lateral current distribution at the active layer is a known, explicit functional form that depends only on the structure of the device. We have approximated these functions by a supergaussian in the case of bottom-emitting devices, while a ring-shaped current distribution has been taken for top-emitting devices. Similarly, the radial dependence of the excess refractive index $\Delta n(r)$ has been assumed to be parabolic, allowing the effects of thermal lensing in the device to be analyzed. Although the electrical and thermal models have not been yet implemented, they might be included in our optical model in order to self-consistently determine the distribution in current density, temperature and optical field. The threshold characteristics of top- and bottom-emitting VCSELs have been analyzed by using an analytical approximation to the optical susceptibility of the QW media that allows a frequency-dependent gain and refractive index spectra to be incorporated into the VCSEL dynamics.

The threshold behavior, threshold current, and mode selection of the transverse modes have been systematically discussed in a semi-analytical way for both devices, for different thermal lensing strengths and detunings. The interplay of the lateral confinement factor and material gain spectrum in the selection mechanisms have been explored in detail. Low to moderate values of the thermal lensing are required in order to maintain single-mode operation since, for strong thermal lensing, the confinement of all the modes increases while their frequency spacing is not so affected, hence leading to poor mode discrimination at threshold. VCSELs with homogeneous current injection select the fundamental transverse mode at threshold unless the cavity resonance is strongly detuned to the red side of the gain peak. However, VCSELs with ring shaped current profiles allow for selecting different transverse modes depending on the strength of the thermal lens. In this case, the overlap of the mode profile with the ring-shaped carrier density dominates for the mode selection at threshold. These semi-analytical predictions have been corroborated by close-to-threshold numerical simulations of the spatially-extended VCSEL. The evolution of the near fields has been followed, demonstrating that the laser onset of a top emitting VCSEL may be initiated from a higher-order transverse mode. Moreover, from our results we infer the validity and usefulness of a modal expansion in terms of modal profiles and frequencies, demonstrating that in the limit of very weak guidance the carrier-induced gain and refractive index strongly modify the mode characteristics. Finally, we have analyzed the dynamical response of the VCSEL to a current pulse of short duration. This approach has simplified considerably the analysis because the temperature distribution is approximately stationary during the 
pulse. By analyzing the response of VCSELs to a current pulse, we have demonstrated a clear tendency toward multimode emission even in the case of very weak TL, which may affect the performance of the device in data-transmission applications because of an enhanced pulse dispersion, but that lowers the degree of spatial coherence of the beam profile and, therefore, the modal noise in multimode-fiber optical links. In order to enhance single-mode operation, cavity detunings on the blue side of the gain peak would be recommended, although in this case there would be an increase in threshold current. Moreover, the thermal shift of the cavity mode as the current is increased would reduce the operating range through thermal rolloff.

\section{APPENDIX}

The equations (36) are integrated by implementing a spectral method that treats the linear terms exactly, while the nonlinear terms are integrated to within an accuracy $o(d t)$. Formally, (36) can be expressed

$$
\begin{aligned}
\partial_{t} A_{ \pm}\left(\vec{r}_{\perp}, t\right) & =\hat{\mathcal{M}} A_{ \pm}+\mathcal{N}\left(\vec{r}_{\perp}, t\right)+\sqrt{\beta D_{ \pm}} \xi_{ \pm}\left(\vec{r}_{\perp} ; t\right) \\
\hat{\mathcal{M}} & =-\kappa+\frac{i c^{2}}{2 \Omega n_{e} n_{g}} \nabla_{\perp}^{2}
\end{aligned}
$$

$\hat{\mathcal{M}}$ being a position and time-independent linear operator and $\mathcal{N}\left(\vec{r}_{\perp}, t\right)$ containing the remaining terms of (36). One starts the numerical integration from initial conditions for $A_{ \pm}\left(\vec{r}_{\perp}, 0\right)$, taken as spontaneous emission distributions, and $D_{ \pm}\left(\vec{r}_{\perp}, 0\right)$ that corresponds to the "off" state [see (38)]. The next step is to self-consistently obtain the operation "frequency" $z_{ \pm}\left(\vec{r}_{\perp}, 0\right)$, with $z_{ \pm}\left(\vec{r}_{\perp}, t\right) \equiv \partial_{t} A_{ \pm} / A_{ \pm}$. From (36), we have

$$
\begin{aligned}
z_{ \pm} & =-\kappa+\frac{i a \Gamma}{2} \chi_{ \pm}\left(\Omega+i z_{ \pm}, D_{+}, D_{-}\right)+\mathcal{G}\left(\vec{r}_{\perp}, t\right) \\
\mathcal{G}\left(\vec{r}_{\perp}, t\right) & =\frac{1}{A_{ \pm}}\left[i \hat{\mathcal{L}} A_{ \pm}-\left(\gamma_{a}+i \gamma_{p}\right) A_{\mp}\right] .
\end{aligned}
$$

At each spatial point, for known values of $A_{ \pm}\left(\vec{r}_{\perp}, t\right)$ and $D_{ \pm}\left(\vec{r}_{\perp}, t\right),(52)$ has to be solved using Newton-Raphson iteration to obtain $z_{ \pm}\left(\vec{r}_{\perp}, t\right)$. From this procedure, we have perfect knowledge of the nonlinear term $\mathcal{N}\left(\vec{r}_{\perp}, t\right)$ of the right-hand side of (52). Following the approach used in [53], the field variables are updated one time step in the Fourier space

$$
\begin{aligned}
\tilde{A}_{q \pm}(t+\Delta t) & =e^{-\alpha_{q} \Delta t} \tilde{A}_{q \pm}(t)+\frac{1-e^{-\alpha_{q} \Delta t}}{\alpha_{q}} \tilde{\mathcal{N}}_{q \pm}(t) \\
& +\sqrt{\beta \frac{\left(1-e^{-2 \operatorname{Re} \alpha_{q} \Delta t}\right)}{2 \operatorname{Re} \alpha_{q}}} \tilde{\Psi}_{q \pm}(t)+o\left(\Delta t^{2}\right)
\end{aligned}
$$

where $q=\left(q_{x}, q_{y}\right) . \tilde{A}_{q \pm}, \alpha_{q} \equiv \kappa-i c^{2} /\left(2 \Omega n_{e} n_{g}\right) q^{2}$, and $\tilde{\mathcal{N}}_{q \pm}(t)$ represent the discrete Fourier components (FFT) of $A_{ \pm}\left(\vec{r}_{\perp}, t\right), \hat{\mathcal{M}}$, and $\mathcal{N}\left(\vec{r}_{\perp}, t\right)$ respectively. $\Psi_{q \pm}(t)$ represents a white noise contribution at a transverse wavevector $q$ obtained by Fourier transforming in space

$$
\tilde{\Psi}_{q \pm}(t)=\mathcal{F} \mathcal{F} \mathcal{T}\left\{\sqrt{D_{ \pm}\left(\vec{r}_{\perp}, t\right)} \xi_{ \pm}\left(\vec{r}_{\perp} ; t\right)\right\} .
$$

Once the field variables have been updated, the carrier equations, being the slow variables in the problem, are integrated using an Euler method. The diffusion terms in (37), involving terms like $\nabla_{\perp}^{2} D_{ \pm}$, are calculated in the Fourier space.

\section{ACKNOWLEDGMENT}

The authors thank Prof. M. San Miguel for his useful discussions, and a critical reading of the manuscript.

\section{REFERENCES}

[1] T. Ackemann, S. Barland, M. Cara, S. Balle, J. R. Tredicce, R. Jäger, M. Grabherr, M. Miller, and K. J. Ebeling, "Spatial mode structure of bottom-emitting broad-area vertical-cavity surface-emitting lasers," $J$. Opt. Soc. Amer. B, vol. 2, pp. 406-412, 2000.

[2] M. San Miguel, "Polarization properties of vertical-cavity surface-emitting lasers," in Semiconductor Quantum Optoelectronics. Bristol, U.K.: Institute of Physics, 1999, p. 339.

[3] K. D. Choquette, D. A. Richie, and R. A. Leibenguth, "Temperature dependence of gain-guided vertical-cavity surface-emitting laser polarization," Appl. Phys. Lett., vol. 69, pp. 2062-2064, 1994.

[4] K. D. Choquette, R. P. Schneider, K. L. Lear, and R. A. Leibenguth, "Gain-dependent polarization properties of vertical-cavity lasers," IEEE J. Select. Topics Quantum. Electron., vol. 1, pp. 661-666, 1995.

[5] K. Panajotov, B. Ryvkin, J. Danckaert, M. Peeters, H. Thienpoint, and I. Veretennicoff, "Polarization switching in VCSEL's due to thermal lensing," IEEE Photon. Technol. Lett., vol. 10, pp. 6-8, Jan. 1998.

[6] B. Ryvkin, K. Panajotov, A. Georgievski, J. Danckaert, M. Peeters, G. Verschaffelt, H. Thienpoint, and I. Veretennicoff, "Effect of photon-energy-dependent loss and gain mechanisms on polarization switching in vertical-cavity surface-emitting lasers," J. Opt. Soc. Amer. B, vol. 16, pp. 2106-2113, 1999.

[7] M. San Miguel, Q. Feng, and J. V. Moloney, "Light-polarization dynamics in surface-emitting semiconductor lasers," Phys. Rev. A, vol. 52, pp. 1728-1739, 1995.

[8] J. Mulet, C. R. Mirasso, and M. San Miguel, "Polarization resolved intensity noise in vertical-cavity surface-emitting lasers," Phys. Rev. A, vol. 64 , p. $023817,2001$.

[9] D. Burak, J. V. Moloney, and R. Binder, "Macroscopic versus microscopic description of polarization properties of optically anisotropic vertical-cavity surface-emitting lasers," IEEE J. Quantum Electron., vol. 36, pp. 956-970, 2000.

[10] S. Balle, E. Tolkachova, M. San Miguel, J. R. Tredicce, J. Martín-Regalado, and A. Gahl, "Mechanisms of polarization switching in singletransverse-mode VCSELs: Thermal shift and nonlinear semiconductor dynamics," Opt. Lett., vol. 24, pp. 1121-1123, 1999.

[11] M. San Miguel, S. Balle, J. Mulet, C. R. Mirasso, E. Tolkachova, and J. R. Tredicce, "Combined effects of semiconductor gain dynamics, spin dynamics and thermal shift in polarization selection in VCSELs," Proc. SPIE, vol. 3944, pp. 242-251, 2000.

[12] G. R. Hadley, K. L. Lear, M. E. Warren, K. D. Choquette, J. W. Scott, and S. W. Corzine, "Comprehensive numerical modeling of verticalcavity surface-emitting lasers," IEEE J. Quantum Electron., vol. 32, pp. 607-616, 1996.

[13] D. Burak and R. Binder, "Cold-cavity vectorial eigenmodes of VCSEL's,” IEEE J. Quantum Electron., vol. 33, pp. 1205-1215, 1997.

[14] H. Wenzel and H.-J. Wünsche, "The effective frequency method in the analysis of vertical-cavity surface-emitting lasers," IEEE J. Quantum Electron., vol. 33, pp. 1156-1162, 1997.

[15] M. J. Noble, J. P. Loehr, and J. A. Lott, "Analysis of microcavity VCSEL lasing modes using a full-vectorial weighted index method," IEEE J. Quantum Electron., vol. 34, pp. 1890-1903, 1998.

[16] B. Demeulenaere, P. Bienstman, B. Dhoedt, and R. G. Baets, "Detailed study of the AlAs-oxidized apertures in VCSEL cavities for optimized modal performance," IEEE J. Quantum Electron., vol. 35, pp. 358-367, 1999.

[17] L. Raddatz, I. H. White, H. D. Summers, K. H. Hahn, M. R. Tan, and S.-Y. Wang, "Measurement of guiding effects in vertical-cavity surfaceemitting lasers," IEEE Photon. Technol. Lett., vol. 8, pp. 743-745, 1996.

[18] W. Nakwaski and M. Osinski, "Thermal properties of vertical-cavity surface-emitting lasers," in Progress in Optics XXXVIIII. Amsterdam, The Netherlands: Elsevier North-Holland, 1998, pp. 165-262.

[19] A. Valle, J. Sarma, and K. A. Shore, "Spatial holeburning effects on the dynamics of vertical-cavity surface-emitting lasers," IEEE J. Quantum Electron., vol. 31, pp. 1423-1431, 1995. 
[20] J. Law and G. P. Agrawal, "Mode-partition noise in vertical-cavity surface-emitting lasers," IEEE Photon. Technol. Lett., vol. 9, pp. 437-439, 1997.

[21] J. Martín-Regalado, S. Balle, and M. San Miguel, "Polarization and transverse mode dynamics of gain-guided vertical-cavity surface-emitting lasers," Opt. Lett., vol. 22, pp. 460-462, 1997.

[22] J. Martín-Regalado, S. Balle, M. San Miguel, A. Valle, and L. Pesquera, "Polarization and transverse mode selection in quantum-well verticalcavity surface-emitting lasers: Index- and gain-guided devices," J. Opt. B: Quantum Semiclass. Opt., vol. 9, pp. 713-736, 1997.

[23] T. Rössler, R. A. Indik, G. K. Harkness, J. V. Moloney, and C. Z. Ning, "Modeling the interplay of thermal effects and transverse mode behavio in native-oxide-confined vertical-cavity surface-emitting lasers," Phys. Rev. A, vol. 58, pp. 3279-3292, 1998 .

[24] C. Z. Ning and P. M. Goorjian, "Microscopic modeling and simulation of transverse-mode dynamics of vertical-cavity surface-emitting lasers," J. Opt. Soc. Amer. B, vol. 16, pp. 2072-2082, 1999.

[25] H. Haug and S. Schmitt-Rink, "Electron theory of the optical properties of laser excited semiconductors," Prog. Quantum Electron., vol. 9, p. 3, 1984.

[26] M. Lindberg and S. W. Koch, "Effective bloch equations for semiconductors," Phys. Rev. B, vol. 38, pp. 3342-3350, 1988

[27] M. Lindberg, S. An, S. W. Koch, and M. Sargent, III, "Strong-field modulation of semiconductor luminescence spectra," Phys. Rev. A, vol. 40, pp. 4415-4425, 1989.

[28] H. Haug and S. W. Koch, "Semiconductor laser theory with many-body effects," Phys. Rev. A, vol. 39, pp. 1887-1898, 1989.

[29] D. Burak, J. V. Moloney, and R. Binder, "Microscopic theory of polarization properties of optically anisotropic VCSELs," Phys. Rev. A, vol. 61, pp. 53809-53830, 2000.

[30] S. Balle, "Simple analytical approximations for the gain and refractive index spectra in quantum-well lasers," Phys. Rev. A, vol. 57, pp. 1304-1312, 1998

[31] C. M. Bowden and G. P. Agrawal, "Generalized Bloch-Maxwell formulation for semiconductor lasers," Opt. Commun., vol. 100, pp. 147-152, 1993.

[32] S. Balle, "Effective two-level model with asymmetric gain for laser diodes," Opt. Commun., vol. 119, pp. 227-235, 1995.

[33] C. Z. Ning, R. A. Indik, and J. V. Moloney, "Effective bloch equations for semiconductor lasers and amplifiers," IEEE J. Quantum Electron., vol. 33, pp. 1543-1550, 1997.

[34] A. K. J. van Doorn, M. P. van Exter, and J. P. Woerdman, "Elasto-optic anisotropy and polarization orientation of vertical-cavity surface-emitting semiconductor lasers," Appl. Phys. Lett., vol. 69, pp. 1041-1043, 1996.

[35] M. P. van Exter, A. K. Jansen van Doorn, and J. P. Woerdman, "Electrooptic effect and birefringence in vertical-cavity surface-emitting lasers," Phys. Rev. A, vol. 56, pp. 845-853, 1997.

[36] L. Viña, "Spin relaxation rate in low-dimensional systems," J. Phys.: Condens. Matter, vol. 11, pp. 5929-5952, 1999.

[37] B. Mroziewicz, M. Bugajski, and W. Nakwaski, Physics of Semiconductor Lasers. Amsterdam, The Netherlands: North-Holland, 1991.

[38] M. Sargent, III, M. O. Scully, and W. Lamb, Laser Physics. New York: Addison-Wesley, 1974.

[39] A. Yariv, Optical Electronics. Philadelphia, PA: Holt, Rinehart and Winston, 1991.

[40] G. P. Agrawal and N. K. Dutta, Long-Wavelength Semiconductor Lasers. New York: Van Nostrand-Reinhold, 1986.
[41] L. A. Coldren and S. W. Corzine, Diode Lasers and Photonic Integrated Circuits. New York: Wiley, 1995.

[42] F. Stern, "Calculated spectral dependence of gain in excited GaAs," $J$. Appl. Phys., vol. 47, p. 5382, 1976

[43] D. S. Citrin and Y.-C. Chang, "Theory of gain in quantum-wire lasers grown in V-grooves," IEEE J. Quantum Electron., vol. 29, pp. 97-108, 1993.

[44] T. Yamanaka, Y. Yoshikuni, K. Yokoyama, W. Lui, and S. Seki, "Theoretical study on enhanced differential gain and extremely reduced linewidth enhancement factor in quantum-well lasers," IEEE J. Quantum Electron., vol. 29, pp. 1609-1616, 1993.

[45] W. L. Li, Y. K. Su, and D. H. Jaw, "The influences of refractive index dispersion on the modal gain of a quantum-well laser," IEEE J. Quantum Electron., vol. 33, pp. 416-423, 1997.

[46] P. M. Enders, "Enhancement and spectral shift of optical gain in semiconductors from non-Markovian intraband relaxation," IEEE $J$. Quantum Electron., vol. 33, pp. 580-588, 1997.

[47] W. W. Chow, S. W. Koch, and M. Sargent, III, Semiconductor Laser Physics. Berlin, Germany: Springer-Verlag, 1994

[48] O. Hess and T. Kuhn, "Maxwell-bloch equations for spatially inhomogeneous semiconductor lasers. I. Theoretical formulation," Phys. Rev. A, vol. 54, pp. 3347-3359, 3360-3368, 1996.

[49] T. Makino, "Analytical formulas for the optical gain of quantum wells," IEEE J. Quantum Electron., vol. 32, pp. 493-501, 1996.

[50] A. W. Synder and J. D. Love, Optical Waveguide Theory. New York: Chapman and Hall, 1983.

[51] N. K. Dutta, "Analysis of current spreading, carrier diffusion, and transverse mode guiding in surface emitting lasers," J. Appl. Phys., vol. 65 , pp. 1961-1963, 1990

[52] W. Nakwaski and R. P. Sarzala, "Transverse modes in gain-guided vertical-cavity surface-emitting lasers," Opt. Commun., vol. 148, pp. 63-39, 1998.

[53] R. Montagne, E. Hernández-García, A. Amengual, and M. San Miguel, "Wound-up phase turbulence in the complex Ginzburg-Landau equation," Phys. Rev. E, vol. 56, pp. 151-167, 1997.

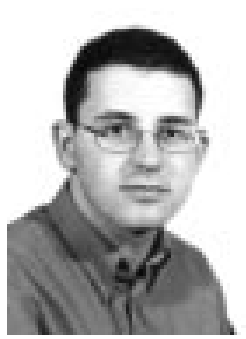

Josep Mulet was born in Lloseta, Mallorca, Spain, in 1975 . He is currently working toward the Ph.D. degree in physics at the University of the Balearic Islands (UIB), Palma de Mallorca, Spain.

His research topics include modeling and dynamics of VCSELs, dynamics of semiconductor lasers with external optical feedback, and synchronization of chaotic semiconductor lasers.

Salvador Balle was born in Manacor, Mallorca, Spain, in 1961. He received the Ph.D. degree in physics from the Universitat Autònoma de Barcelona, Barcelona, Spain, in 1988.

His research interests are the nonlinear dynamics of semiconductor lasers and amplifiers and the modeling of the nonlinear optical properties of these devices. 